\title{
Growth functions on Fuchsian groups and the Euler characteristic
}

\author{
William J. Floyd ${ }^{1, \star}$ and Steven P. Plotnick ${ }^{2, \star \star}$ \\ ${ }^{1}$ Department of Mathematics, University of Michigan, Ann Arbor, MI 48109, USA, \\ and Department of Mathematics, VPI \& SU, Blacksburg, VA 24061, USA \\ ${ }^{2}$ Department of Mathematics, Columbia University, New York, NY 10027, USA
}

Let $G$ be a finitely generated group, and $\Sigma$ a finite generating set for $G$. Then $\Sigma$ determines a norm on $G$, called the word norm, defined by letting $|g|$ be the minimal length of $g$ as a word in $\Sigma$. Following Milnor [15], we define the growth series $g(z)$ $=1+a_{2} z+\ldots+a_{n} z^{n} \ldots$, where $a_{n}$ is the number of elements of $G$ of word norm exactly $n$. Much of the early work (e.g., $[1,16,17,23])$ on growth series was concerned with asymptotic properties of the coefficients of $g(z)$. This work culminated in Gromov's theorem [12] that $G$ has polynomial growth if and only if it is virtually nilpotent and in Grigorchuk's theorem [11] that there are finitely generated groups whose growth is neither polynomial nor exponential.

If $G$ is a Coxeter group and $\Sigma$ is the standard generating set for $G$, Bourbaki [3] showed that the growth series $g(z)$ is the series of a rational function $f(z)$ and Serre [20] showed that $f(1)=1 / \chi(G)$, where $\chi(G)$ is the rational Euler characteristic of $G$. Note that if $G$ is finite then $g(1)=\operatorname{order}(G)=1 / \chi(G)$, but $g(z)$ is not defined at one if $G$ is infinite. If $G$ is a compact hyperbolic or irreducible Euclidean Coxeter group and $\Sigma$ is the standard generating set for $G$, Serre showed that $f(1 / z)= \pm f(z)$, and so the poles of $f$ are algebraic units. In contrast to the work on the asymptotic properties of the coefficients of $g(z)$, these results raised the possibility of a beautiful theory of the exact structure of growth functions on groups. However, a decade went by before there was much further work on this subject.

In two papers ([5] and [6]) Cannon studied growth functions for cocompact hyperbolic groups. If $G$ is a cocompact hyperbolic group and $\Sigma$ is any finite generating set for $G$, he showed that the coefficients $a_{n}$ of the growth series $g(z)$ satisfy a linear recursion, and hence $g(z)$ is the power series of a rational function $f(z)$. He also computed some examples when $G$ is a closed surface group or a compact hyperbolic Coxeter group. If $G$ is a closed surface group and $\Sigma$ is a geometric generating set (see below for a definition) for $G$ coming from a one relator presentation, then $f$ is reciprocal $(f(z)=f(1 / z)), f(1)=1 / \chi(G)$, the 
numerator of $f$ is a product of cyclotomic polynomials, and the denominator of $f$ is the product of a Salem polynomial and cyclotomic polynomials.

In 1983, Benson [2] showed that if $G$ is a finite extension of $\mathbb{Z}^{n}$ and $\Sigma$ is any finite generating set for $G$, then the growth series $g(z)$ is the series of a rational function $f(z)$, and $f$ has a pole of order $n$ at $z=1$. Since these groups have Euler characteristic $\chi(G)=0$, this fits in with the conjecture that $f(1)=1 / \chi(G)$ when the power series $g(z)$ is the series of a rational function $f$ and when $\chi(G)$ is defined.

Smythe [21] studied the relation between $f(1)$ and $\chi(G)$ by thinking of $G$ as a filtered semigroup and studying $R G$-resolutions of $R$ (where $R$ is the integers or a field). While he gets conditions for which $f(1)=1 / \chi(G)$, it seems to be very hard to work with these conditions.

There have been further calculations (e.g., [10] and [22]) to support the conjecture that $f(1)=1 / \chi(G)$. However, a recent example due to Parry [18] shows that this conjecture is not true for all finite generating sets. Let $G=\mathbb{Z} * \mathbb{Z}$ with generators $x$ and $y, \Sigma=\left\{x^{ \pm 2}, x^{ \pm 3}, y^{ \pm 2}, y^{ \pm 3}\right\}$, and $\Sigma^{\prime}=\left\{g \in G:|g|_{\Sigma}<3\right\}$. Parry shows that the growth function of $G$ with respect to the generating set $\Sigma^{\prime}$ is $f(z)$ $=\left(1+24 z-4 z^{2}\right) /\left(1-32 z+4 z^{2}\right)$. Note that $f(1)=-7 / 9$ and $\chi(G)=-1$. As a result of this example, we decided to look at the case when $\Sigma$ is a geometric generating set.

Let $G$ be a discrete group of isometries of hyperbolic $n$-space $\mathbb{H}^{n}$ such that $\mathbb{H}^{n} / G$ has finite volume. Choose a basepoint $x$ in $\mathbb{H}^{n}$ which is not fixed by any non-trivial element of $G$, and let $D=D_{x}=\left\{y \in \mathbb{H}^{n}: d(y, x) \leqq d(y, g x)\right.$ for all $\left.g \in G\right\}$. Then $D$ is a finite sided polyhedron in $\mathbb{H}^{n}$ which is a fundamental region for the action of $G$ on $\mathbb{H}^{n} . D$ is called a Dirichlet region, and the number of sides of $D$ depends on the choice of basepoint $x$. The set of face-pairing elements $\Sigma=\{g \in G: g D \cap D$ has dimension $n-1\}$ is a finite generating set for $G$. If $G$ is finitely generated, we will call a finite generating set $\Sigma$ for $G$ geometric if there is a representation of $G$ as a discrete group of isometries of hyperbolic space $\mathbb{H}^{n}$ so that $\mathbb{H}^{n} / G$ has finite volume and a basepoint $x \in \mathbb{H}^{n}$ so that $\Sigma$ is the set of face pairing elements for $D_{x}$.

Let $G$ be a finitely generated, discrete group of isometries of $\mathbb{H}^{2}$ with $\mathbb{H}^{2} / G$ of finite area and $\Sigma$ a geometric generating set for $G$ with respect to the Dirichlet region $D=D_{x}$. For convenience we also assume that $\Sigma$ has no elements of order two. In $\S 1$ we study the combinatorics of the tessellation of $\mathbb{H}^{2}$ by $\{g D: g \in G\}$. Using this we show: (1) $B(n)=\cup\{g D:|g| \leqq n\}$, the "combinatorial ball of radius $n$ ", is a topological ball, and (2) there is a square matrix $\mathbf{A}$ and a column vector $\mathbf{v}$ so that $g(z)$ is the series of the rational function $f(z)=1+(1 \ldots 1)(\mathbf{I}-z \mathbf{A})^{-1} z \mathbf{v}$. This is motivated by Cannon's proof that $g(z)$ is the series of a rational function, but we may have a different recursion matrix $\mathbf{A}$ and initial vector $\mathbf{v}$.

These facts are used in $\S 2$, where we prove our main result. Loosely speaking, it may be stated as follows:

Theorem 1. Given a Fuchsian group $G$, Dirichlet region $D$, generating set $\Sigma$, and growth function $f(z)$ as above, there is a row vector $\mathbf{w}$ (defined geometrically in terms of D) so that

$$
f(z)=1 / \chi(G)+(1-z)(2 \chi(G))^{-1} \mathbf{w}(\mathbf{I}-z \mathbf{A})^{-1} \mathbf{v} .
$$

Thus $f(1)=1 / \chi(G)$ if $\mathbf{I}-\mathbf{A}$ is invertible. 
From this one would expect that $f(1)=1 / \chi(G)$ for most but not all choices of geometric generating sets for Fuchsian groups. In $\S 3$ we give some examples to illustrate this. We first compute the growth functions associated to tilings $\mathbb{H}^{2}$ by (even-sided) regular polygons, with all angles $2 \pi / p, p \geqq 3$. In these cases, it turns out that $\mathbf{I}-\mathbf{A}$ is invertible, so $f(1)=1 / \chi$. We also give an example, on a genus two surface with a less symmetric Dirichlet region, where $\mathbf{I}-\mathbf{A}$ is not invertible but nevertheless $f(1)=1 / \chi$. Finally, we give an example where $\mathbf{I}-\mathbf{A}$ is not invertible and $f(1) \neq 1 / \chi$. Our example has genus 4 and 30 sides, with angles alternating between $2 \pi / 3$ and $2 \pi / 5$. We conclude, in $\S 4$, with an indication of how our methods should extend to higher even-dimensional hyperbolic groups, and with some open problems.

\section{§1. Combinatorics of planar tessellations}

Let $G$ be a finitely generated, discrete group of isometries of either the hyperbolic plane (in which case we assume $\mathrm{H}^{2} / G$ has finite area), the Euclidean plane (in which case we assume $\mathbb{E}^{2} / G$ is compact), or the round sphere $S^{2}$, and let $\Sigma$ be a geometric generating set for $G$ with respect to the Dirichlet region $D=D_{x}$. For each integer $n \geqq 0$ let $B(n)=$ closure $\left(\cup\left\{g D:|g|_{\Sigma} \leqq n\right\}\right)$, where in the first case we are using the Poincare disc model for $\mathbb{H}^{2}$ and taking the closure in $\mathbb{R}^{2}$ (the closure is adding the parabolic points at infinity). One can think of $B(n)$ as the "combinatorial ball of radius $n$ " around $x$.

Our goal in this section is to give an inductive construction of the group graph of $G$ with generators $\Sigma$. As consequences of this construction, we will show:

(1) If $G$ is a group of hyperbolic or Euclidean isometries, then $B(n)$ is a topological ball, and the cells $g D$ with $|g|_{\Sigma}=n+1$ intersect $B(n)$ and each other in rather simple configurations.

(2) The growth series of $G$ with respect to $\Sigma$ is the series of a rational function.

We make one rather mild assumption on $\Sigma$, more out of convenience than necessity - namely, we assume that $\Sigma$ has no elements of order two. Thus, our discussion is essentially disjoint from the case of Coxeter groups, where all elements of $\Sigma$ have order two.

We first describe how the Dirichlet region determines a presentation for $G$ with generators $\Sigma$. For simplicity, first assume that $D$ is compact and $G$ is torsion-free, so that $G$ is the fundamental group of a closed surface. Let $V=\{$ vertices $v$ of $D$, and put an equivalence relation $\sim$ on $V$ by $v \sim w$ if there is a finite sequence $v=v_{0}$, $v_{1}, \ldots, v_{n}=w$ of vertices of $D$ and elements $g_{1}, \ldots, g_{n} \in \Sigma$ with $g_{j} v_{j-1}=v_{j}$ for all $j \in\{1, \ldots, n\}$ (so each equivalence class corresponds to one vertex in the quotient surface). Each equivalence class of vertices is known as a vertex cycle. Let $\left\{v_{1}, \ldots, v_{m}\right\}$ consist of one vertex from each equivalence class. For each $i \in\{1, \ldots, m\}$, let $c_{i}$ be the word in $\Sigma$ determined by an edge-path in the 1 -skeleton of the dual tessellation which traverses the link of $v_{i}$. If $a_{0}=v_{i}, a_{1}=g_{1} a_{0}, a_{2}$ $=g_{2} a_{1}, \ldots, a_{r}=g_{r} a_{r-1}, a_{0}=g_{r+1} a_{r}$ is a minimal listing (i.e., $a_{j} \neq a_{i}$ if $i \neq j$ ) of the vertex cycle through $v_{i}$, then the corresponding word is $g_{r+1} g_{r} \ldots g_{2} g_{1}$. Note that choosing a different initial edge for the edge-path would give a cyclic permutation of $c_{i}$ and changing the orientation of the edge-path would replace $c_{i}$ by its inverse. Then $G$ has presentation $\left\langle\Sigma \mid c_{1}, \ldots, c_{m}\right\rangle$. We give an example in Fig. 1. 


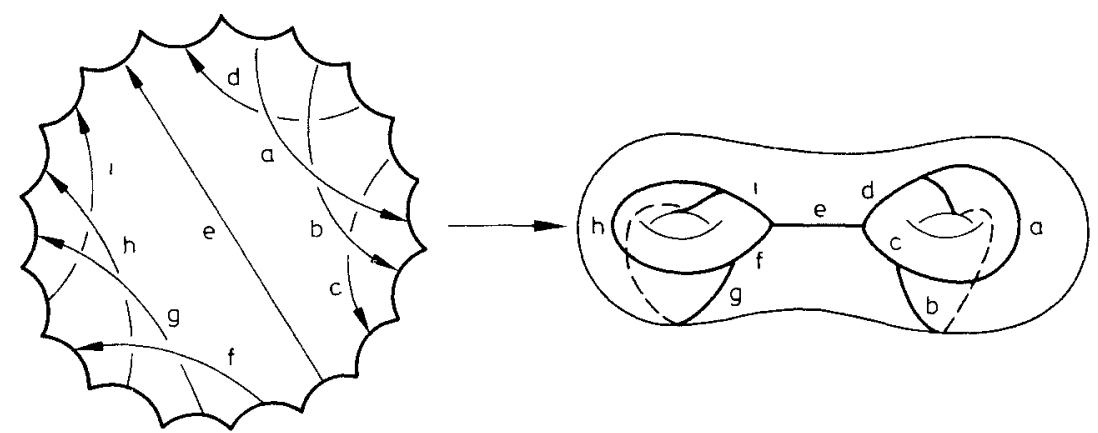

Fig. 1. $G \cong\left\langle a, b, c, d, e, f, g, h, i \mid a b^{-1} d, b c^{-1} a^{-1}, c e d^{-1}, f i e^{-1}, g h^{-1} f^{-1}, h i^{-1} g^{-1}\right\rangle$

In the general case, $D$ may be non-compact and $G$ may have torsion. Recall that $\Sigma$ has no elements of order two. In particular, $\Sigma$ contains no reflections, so each element of $\Sigma$ pairs two different edges. Hence, $D$ is an even-sided polygon in $\mathbb{H}^{2}$, $\mathbb{E}^{2}$, or $S^{2}$, possibly with some vertices at $\infty$ in the hyperbolic case. As before, we follow the edge pairings to compute the vertex cycles and words $c_{1}, \ldots, c_{m}$. We also assign an integer $n_{i} \in\{1,2, \ldots, \infty\}$ to each cycle as follows: Given $i \in\{1, \ldots, m\}$, add the (interior) angles in $D$ at each vertex of the cycle through $v_{i}$. This gives the total angle at the image of $v_{i}$, say $\bar{v}_{i}$, in the quotient $\mathbb{H}^{2} / G, \mathbb{E}^{2} / G$, or $S^{2} / G$. Since $G$ is discrete, this angle must have the form $2 \pi / n_{i}$ for some positive integer $n_{i}$. If $n_{i}=1$, the quotient has a non-singular geometric structure near $\bar{v}_{i}$, and $c_{i}$ has order 1 in $G$. If $1<n_{i}<\infty, \bar{v}_{i}$ is a branch point of index $n_{i}$, and $c_{i}$ is an elliptic element of order $n_{i}$. If $n_{i}=\infty$, the cycle through $v_{i}$ consists of vertices at $\infty$, neighbourhoods of the vertices in the cycle are glued together to form a cusp in $\mathrm{H}^{2} / G$, and $c_{i}$ is a parabolic element of infinite order. Then $G$ has the presentation $\left\langle\Sigma \mid\left(c_{1}\right)^{n_{1}}, \ldots,\left(c_{m}\right)^{n_{m}}\right\rangle$, where infinitely long relators, of course, may be deleted.

We now describe the possibilities for the "spine" of the quotient; that is, the image of $\partial D$. In the torsion-free, cocompact case, the spine is a finite graph, and every vertex has valence at least three, as in Fig. 1. In the presence of torsion, there may be vertices of valence one or two. For example, the orientation-preserving subgroup of a triangle group can give valence one or two vertices, as shown in Fig. 2. Finally, if $\mathbb{H}^{2} / G$ is not compact, the spine might be disconnected, as shown in Fig. 3.

We now describe the properties that we need of the words $c_{1}, \ldots, c_{m}$ to inductively construct the group graph. Let $C=\left\{c_{1}, \ldots, c_{m}\right\}$. For each $c_{i}$, let $\left[c_{i}\right]$ consist of all cyclic permutations of $c_{i}$, and let $W=\cup\left\{c^{n_{i}}: c \in\left[c_{i}\right]\right.$ and $\left.i \in\{1, \ldots, m\}\right\}$. By convention, if $n^{i}=\infty, c^{n_{i}}$ means the bi-infinite word ...ccc...; if $g$ is the first letter of $c$, we say $c^{\infty}$ begins with $g$. Note that, since $\Sigma$ has no element of order two, every element of $W$ has length at least three.

Lemma 1 (compare Cannon [6, Lemma 3.2]). (i) The elements of $C$ are cyclically reduced.

(ii) For each $g \in \Sigma, g^{ \pm 1}$ appears exactly twice in the elements of $C$.

(iii) Elements of $W \cup W^{-1}$ are determined by their first two letters. 


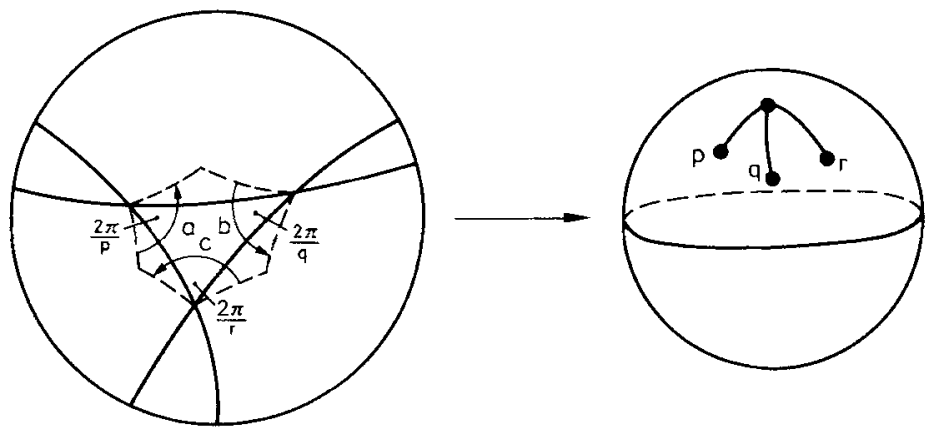

a

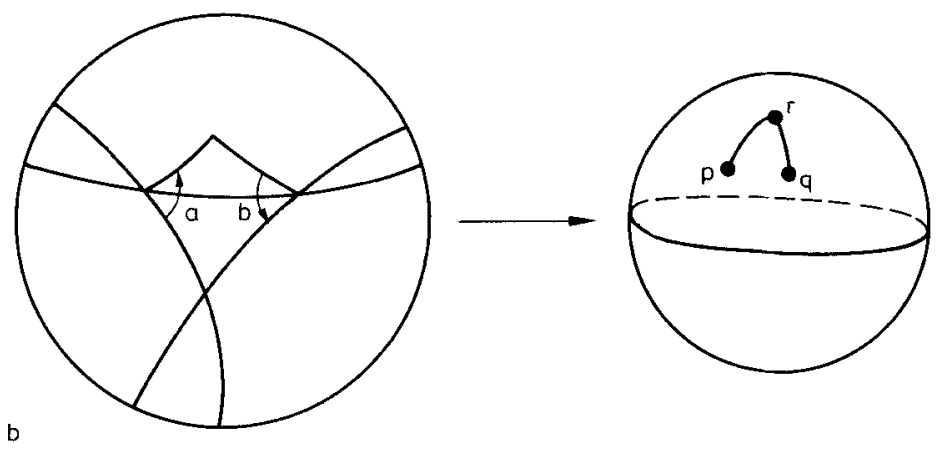

Fig. 2a, b. Two possible "spines" for a $(p, p, r)$-triangle group. a $G \cong\left\langle a, b, c \mid a^{p}, b^{q}, c^{r}, a b c\right\rangle$. b $G \cong\langle a$, $b\left|a^{p}, b^{q},(a b)^{r}\right\rangle$

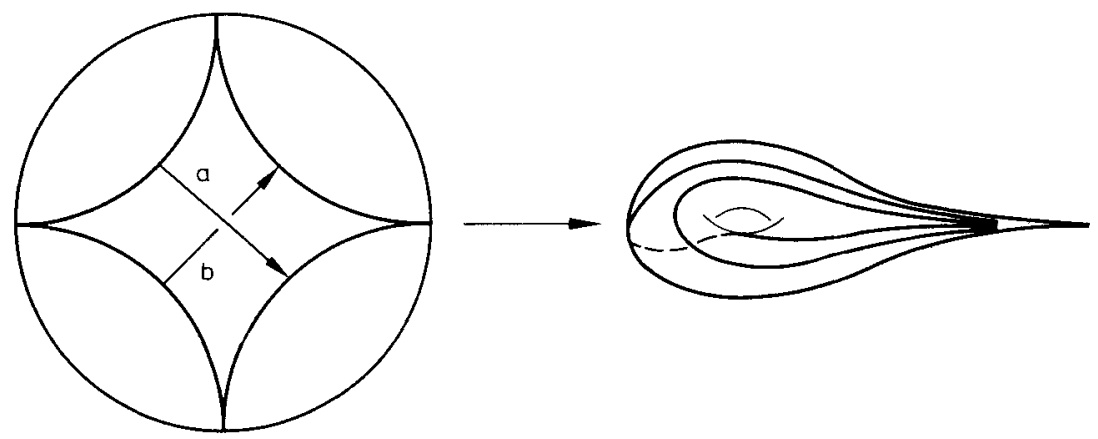

Fig. 3. $G \cong\left\langle a, b \mid\left(a b a^{-1} b^{-1}\right)^{\infty}\right\rangle$

(iv) For each $g \in \Sigma$, exactly two of the elements of $W \cup W^{-1}$ begin with $g$.

(v) If $f, g$ and $h$ are distinct elements of $\Sigma$, then $f g^{-1}, f h$ and $g h$ do not all occur as initial segments of elements of $W \cup W^{-1}$.

Proof. (i) The combination $g g^{-1}$ could not occur for a geometric generating set.

(ii) Let $e$ be the edge $D \cap g D$. Then $g^{ \pm 1}$ occurs exactly once for each end or vertex of $e$. 


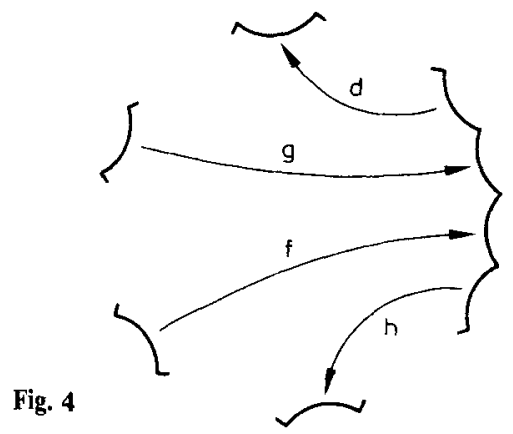

(iii) Let $g \in \Sigma, w \in\left(W \cup W^{-1}\right)$ such that $w$ starts with $g$, and let $e$ be the edge $D \cap g D$. Then $w$ is the word determined by following one of the two vertices or ends of $e$. So there are only two possibilities, and these are detected by the second letter of $w$.

(iv) This follows from (ii).

(v) Let $f, g$ and $h$ be distinct elements of $\Sigma$, and suppose that $f g^{-1}$ and $f h$ are initial segments of elements of $W \cup W^{-1}$. Without loss of generality, we can assume that the edge-pairing elements near the edge $D \cap f D$ are as in Fig. 4. If $g h$ occurs as an initial segment of an element of $W \cup W^{-1}$, then $d=h$ and $D$ only has 3 sides, a contradiction.

Our construction of the group graph follows an argument of Cannon in [6], and we adopt Cannon's notation from [6] and [7]. Given a graph $L$ and a directed edge $e$ in $L$, let $\bar{e}$ be the same edge but with the opposite direction. We say that a graph $L$ is colored by $\Sigma$ if each directed edge $e$ in $L$ has a label $c(e) \in \Sigma$ such that $c(\bar{e})$ $=(c(e))^{-1}$. We call $c(e)$ the color of $e$. If $L$ is colored by $\Sigma$, then each edge-path $\alpha$ in $L$ determines a word $c(\alpha)$ in $\Sigma$ by concatenating the colors of the successive directed edges of the edge-path. A colored graph $L$ is a prepermutation graph if for each vertex $v \in L$ and each color $c$ in $\Sigma$ there is at most one edge emanating from $v$ with color $c$.

Now assume that $G$ is infinite, i.e. either hyperbolic or Euclidean. Let $K \subset \mathbb{H}^{2}$, $\mathbb{E}^{2}$ be the 1-complex with vertices the elements $\{g x: g \in G\}$, and with two vertices $g x, h x$ connected by a geodesic arc if $g D$ and $h D$ intersect in an edge (this occurs exactly when $h g^{-1} \in \Sigma$ ). Since $\Sigma$ has no elements of order two, $K$ is the 1 -skeleton of the dual tessellation. For each vertex $v$ in $K$ and $g \in \Sigma$, there is exactly one directed edge $e$ in $K$ from $v$ to $g v, K$ is colored by $\Sigma$ if we give $e$ the color $g$, and $K$ is exactly the group graph of $G$ with generators $\Sigma$.

Using Lemma 1, we can now give an inductive construction of $K$. Our building blocks are graphs $\Lambda_{\omega}(v)$ which are colored by $\Sigma$, where $\omega \in W$ and $v$ is a vertex. Given a symbol $v$ and a finite word $\omega \in W, \Lambda_{\omega}(v)$ is a cyclic graph such that one vertex of $\Lambda_{\omega}(v)$ is labelled by $v$ and the cyclic edge-loop, based at the vertex labelled $v$, which traverses each edge exactly one time determines the word $\omega$ or $\omega^{-1}$ (depending on the direction). If $v$ is a vertex and $\omega=c^{\infty} \in W$, then $\Lambda_{\omega}(v)$ is an infinite graph homeomorphic to $(-\infty, \infty)$ by a homeomorphism taking the vertex labelled $v$ to 0 . In this case $A_{\omega}(v)$ is colored so that one of the two edge-paths which traverse each edge exactly one time determines the word $c^{\infty}$ and the segment which starts at $v$ 
and has length $|c|$ determines the word $c$. We will construct graphs

$$
\Gamma(0) \subset \Gamma(1) \subset \ldots \subset \Gamma(n) \subset \ldots \subset \bigcup_{n=0}^{\infty} \Gamma(n)=\Gamma
$$

such that $\Gamma(0)$ is a base vertex $v_{0}$, and for all $n>0 \Gamma(n)$ is a connected, prepermutation graph which is obtained from $\Gamma(n-1)$ by adding some $\Lambda_{\omega}(v)$ 's. We will abuse notation and also denote by $\Lambda_{\omega}(v)$ the image of $\Lambda_{\omega}(v)$ in $\Gamma(n)$, since each $\Lambda_{\omega}(v)$ will be embedded. However, it is possible that a $\Lambda_{\omega}$ in $\Gamma(n)$ can have two different base vertices $v_{1}$ and $v_{2}$; that is, the colored graphs $\Lambda_{w_{1}}\left(v_{1}\right)$ and $A_{w_{2}}\left(v_{2}\right)$ are identified in $\Gamma(n)$ even though $v_{1} \neq v_{2}$. (Actually, in very special circumstances, $\Lambda_{\omega}$ may have three base vertices. See Condition 5 below.) We will put norms \|\|$_{n}$ on the $\Gamma(n)$ 's so that if $v$ is a vertex of a $\Lambda_{\omega}$ in $\Gamma(n)$, then $\|v\|_{n}=\min \left\{\|v\|_{n}\right.$ plus the edgepath distance in $A_{\omega}$ from $v$ to $v: v$ is a base vertex for $\left.A_{\omega}\right\}$. Since our norms will have $\|v\|_{i}=\|v\|_{n}$ if $i<n$ and $v \in \Gamma(i)$, we will usually denote $\|v\|_{n}$ by $\|v\|$. Let $V(n-1)$ denote the set of vertices of $\Gamma(n-1)$. For each $n>0$, we require that $\Gamma(n)$ satisfies the following five Conditions:

(1) Each $\Lambda_{\omega}(v)$ of $\Gamma(n)$ is based at a vertex $v \in V(n-1)$.

(2) An edge of $\Gamma(n)$ joining a vertex of norm $\leqq n$ to one of norm $<n$ belongs to exactly two of the $A_{\omega}(v)$ 's in $\Gamma(n)$.

(3) An edge of $\Gamma(n)$ joining two vertices of norm $\geqq n$ belongs to exactly one $A_{\omega}(v)$ in $\Gamma(n)$.

(4) A vertex in $\Gamma(n)$ of norm $n$ is joined by edges to at most two vertices of norm $n-1$. If it is joined to two vertices of norm $n-1$, then all three vertices belong to some finite $\Lambda_{\omega}(v)$ in $\Gamma(n)$.

(5) Except for the case of the orientation-preserving subgroup of a $(3,3, r)$ triangle group, with a 4-sided Dirichlet region as in Fig. 2.b, at most two vertices of a given $\Lambda_{\omega}(v)$ can have equal norm, and if $\Lambda_{\omega}(v)$ contains two vertices of minimal norm, then these vertices are connected by an edge. In this case we say $\Lambda_{\omega}(v)$ is based at both vertices. For the $(3,3, r)$-triangle group, some $\Lambda_{\omega}$ 's are based at three vertices.

Let $\Gamma(0)$ be a vertex $v_{0}$. To construct $\Gamma(1)$, start with the union $\Gamma(0) \amalg \cup\left\{\Lambda_{\omega}\left(v_{0}\right)\right.$ : $\omega \in W\}$, identify all of the vertices labelled $v_{0}$, and call the resulting graph $\Gamma(1)^{\prime}$. For each $g \in \Sigma$, by Lemma 1.iv) there are exactly two directed edges in $\Gamma(1)^{\prime}$ which start at the vertex labelled $v_{0}$ and have color $g$, and these will belong to different $\Lambda_{\omega}$ 's by Lemma 1.i). For each $g \in \Sigma$ identify this pair of directed edges, and call the resulting graph $\Gamma(1)$. Then $\Gamma(1)$ is connected, and $\Gamma(1)$ is a prepermutation graph by Lemma 1.iii). Furthermore, all relators of $\Gamma(1)$ are a consequence of finite elements of $W$, as in Cannon [6]. Put a norm \|\| on the vertices of $\Gamma(1)$ by letting $\|v\|$ be the edge-path distance in $\Gamma(1)$ from the vertex labelled $v_{0}$ to $v$. Note that Conditions 1-5 hold for $\Gamma(1)$, and that each $\Lambda_{w}\left(v_{0}\right)$ is embedded in $\Gamma(1)$.

Now let $n>1$, and assume that $\Gamma(n-1)$ has been constructed satisfying Conditions $1-5$. We will obtain $\Gamma(n)$ from $\Gamma(n-1)$ by adding $\Lambda_{\omega}(v)$ 's for each vertex $v \in \Gamma(n-1)$ with $\|v\|=n-1$. We will first define graphs $\Gamma(v)$ for each $v \in V(n-1)$ with $\|v\|=n-1$, attach these graphs to $\Gamma(n-1)$, and then make whatever edge identifications are required for the result, $\Gamma(n)$, to be a prepermutation graph. 
First assume that $v$ is joined by an edge in $\Gamma(n-1)$ to a unique vertex $v^{\prime} \in \Gamma(n-1)$ with norm $\left\|v^{\prime}\right\|=n-2$. By Condition 2 , the edge $v v^{\prime}$ joining $v$ and $v^{\prime}$ belongs to exactly two $\Lambda_{\omega}(v)$ 's, which we will denote by $\Lambda_{w^{\prime}}(a)$ and $\Lambda_{w^{\prime \prime}}(b)$, in $\Gamma(n-1)$. By Condition 3, at least one of these, which we will assume is $A_{w^{\prime \prime}}(b)$, is not in $\Gamma(n-2)$. It follows that $v^{\prime}$ is a base vertex for $\Lambda_{w^{\prime \prime}}(b)$, and we inductively assume the local picture in $\Gamma(n-1)$ near $v v^{\prime}$ is that of Fig. 5a). Let $w_{1}$ be the cyclic permutation of $w^{\prime}$ which is determined by the edge-loop in $\Lambda_{w^{\prime}}(a)$ which starts at $v$. If $w^{\prime}$ is an infinite word, this means that we read $w^{\prime}$ beginning at $v$. Let $w_{2}$ be the cyclic permutation of $w^{\prime \prime}$ which is determined by the edge-loop in $\Lambda_{w^{\prime \prime}}(v)$ which starts at $v$. Let $W(v)=W-\left\{w_{1}, w_{2}\right\}$. Take $\cup\left\{\Lambda_{\omega}(v): \omega \in W(v)\right\}$, identify all of the vertices labelled $v$, and call the resulting graph $\Gamma(v)^{\prime}$. For each $g \in \Sigma$, there will be at most two directed edges in $\Gamma(v)^{\prime}$ which start at the vertex labelled $v$ and have color $g$. Identify each pair of directed edges from this vertex which have the same color, call the resulting graph $\Gamma(v)$, and note that each $\Lambda_{\omega}(v)$ is embedded in $\Gamma(v)$.

Now assume that $v$ is joined by edges in $\Gamma(n-1)$ to two distinct vertices $x_{1}$ and $x_{2}$ in $\Gamma(n-1)$ with $\left\|x_{1}\right\|=\left\|x_{2}\right\|=n-2$. Then by Condition 4 there is a finite word $w \in W$ and a vertex $x \in \Gamma(n-2)$ with $x, x_{1}$ and $x_{2}$ all in $\Lambda_{w}(x)$. By Conditions 2 and 3 , there are words $w^{\prime}, w^{\prime \prime} \in W$ such that $v \in \Lambda_{w^{\prime}}\left(x_{1}\right), v \in \Lambda_{w^{\prime \prime}}\left(x_{2}\right)$, and both $\Lambda_{w^{\prime}}\left(x_{1}\right)$ and $A_{w^{\prime \prime}}\left(x_{2}\right)$ are in $\Gamma(n-1)$ but not in $\Gamma(n-2)$. Thus, we inductively assume the local picture in $\Gamma(n-1)$ near $v$ is as in Fig. $5 b$. Note that there are no identifications among $A_{w}, A_{w^{\prime}}$, and $\Lambda_{w^{\prime \prime}}$ other than the ones pictured. This is because otherwise either $d_{2}=\left(c_{1}\right)^{-1}, d_{1}=\left(c_{2}\right)^{-1}$, or $d_{1}=d_{2}$. If $d_{2}=\left(c_{1}\right)^{-1}$, then by Lemma 1.iii) the cyclic permutations of $w^{ \pm 1}$ and $\left(w^{\prime \prime}\right)^{ \pm 1}$ determined by the two edge loops which start with $x_{2} v$ must be the same. But this implies that $\Lambda_{w}(x)$ and $\Lambda_{w^{\prime \prime}}\left(x_{2}\right)$ are the same graphs since $\Gamma(n-1)$ is a prepermutation graph, which gives a contradiction. Similar reasoning shows that $d_{2} \neq\left(c_{1}\right)^{-1}$. If $d_{1}=d_{2}$, then there are elements in $W \cup W^{-1}$ beginning $c_{1}\left(c_{2}\right)^{-1}, c_{1} d_{1}$, and $c_{2} d_{2}$, violating Lemma 1.v). Let $w_{1}$ be the cyclic permutation of $w$ determined by the edge-loop in $A_{w}(x)$ which starts at $v$, let $w_{2}$ be the cyclic permutation of $w^{\prime}$ determined by the edge-loop in $\Lambda_{w^{\prime}}\left(x_{1}\right)$ which starts at $v$, and let $w_{3}$ be the cyclic permutation of $w^{\prime \prime}$ determined by the edge-loop in $\Lambda_{w^{\prime \prime}}\left(x_{2}\right)$ which starts at $v$. Let $W(v)=W-\left\{w_{1}, w_{2}, w_{3}\right\}$. Let $\Gamma(v)^{\prime}$ be the graph obtained from $\cup\left\{\Lambda_{w}(v): w \in W(v)\right\}$ by identifying all of the vertices labelled $v$. For each $g \in \Sigma$, there will be at most two directed edges in $\Gamma(v)^{\prime}$ which start at the base vertex labelled $v$ and have color $g$. Identify each pair of directed edges from the base
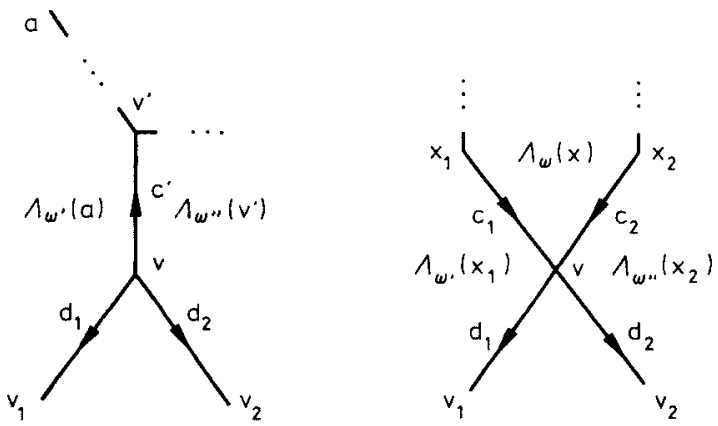

Fig. 5a, b 
Fig. 6

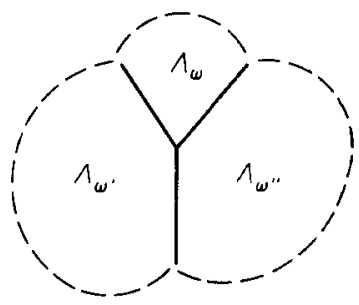

vertex which have the same color, and call the resulting graph $\Gamma(v)$. As before, each $\Lambda_{\omega}(v)$ is embedded in $\Gamma(v)$.

For the future, note that the argument above ruling out $d_{1}=d_{2}$ (see the proof of Lemma $1 . v)$ merely says that one cannot find three $\Lambda_{\omega}$ 's fitting nearly around a vertex, as in Fig. 6. Indeed, since $K$ is the 1-skeleton of the dual tessellation, the link of $v$ would be a copy of $D$, forcing $D$ to be 3 -sided. Similarly, if a future argument forces four $\Lambda_{\omega}$ 's to fit nearly around a vertex, $D$ would be 4-sided.

We will now attach the $\Gamma(v)$ 's to $\Gamma(n-1)$ to obtain $\Gamma(n)$. Let $\Gamma(n)^{\prime}$ be the graph obtained from $\Gamma(n-1)\rfloor \cup\{\Gamma(v): v \in V(n-1)$ and $\|v\|=n-1\}$ by identifying each vertex $v$ in $\Gamma(n-1)$ of norm $n-1$ with the vertex labelled $v$ in $\Gamma(v)$. We will obtain $\Gamma(n)$ as a quotient of $\Gamma(n)^{\prime}$ by further edge identifications, depending on the local structure near the vertices of norm $n-1$ in $\Gamma(n-1)$. Let $v$ be a vertex in $\Gamma(n-1)$ with $\|v\|=n-1$, and let $v_{1}$ and $v_{2}$ be the vertices in $\Gamma(n-1)$ as shown in Fig. 5.a or 5.b (depending on which case $v$ is in). Then each of $\left\|v_{1}\right\|$ and $\left\|v_{2}\right\|$ is either $n-1$ or $n$, depending on the lengths of $w$ and $w^{\prime}$. Identify the pair of directed edges in $\Gamma(n)^{\prime}$ which start at $v$ and have color $d_{1}$, and identify the pair of directed edges in $\Gamma(n)^{\prime}$, which start at $v$ and have color $d_{2}$. Do this for each vertex of norm $n-1$ in $\Gamma(n)^{\prime}$.

We claim that no further identifications need be made near $v$, in order that $\Gamma(n)$ be a prepermutation graph, if $\left\|v_{1}\right\|=\left\|v_{2}\right\|=n$. For, suppose further identifications need be made, involving $v$ and, say, $v_{2}$. Because of the way the $A_{\omega}$ are added to $\Gamma(n-1)$, this can only occur if $v_{2}$ is connected in $\Gamma(n-1)$ to another vertex, $v_{3}$, of norm $n-1$ or $n$, and we must have one of the following two possibilities (Fig. 7):

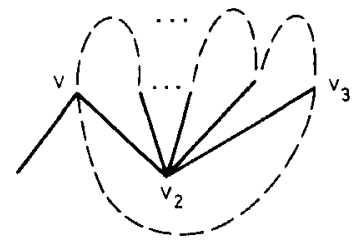

Fig. 7a, b a

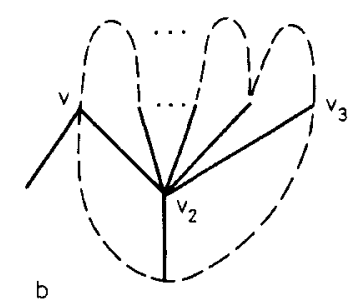

b

Note also that all of the $A_{\omega}$ pictured as lying above $v_{2}$ must already be present in $\Gamma(n-1)$. But then Condition 3 is violated if there are more than one of these. Hence, there is only one, but now the local picture near $v_{2}$ forces $D$ to have two (a) or three (b) sides.

Note also that this argument shows that if some $\Lambda_{\omega} \in \Gamma(n)-\Gamma(n-1)$ is based at more than one vertex (of norm $n-1$ ), then there is an interval in $\Lambda_{\omega}$ connecting 
these two vertices so that every vertex in the interval also has norm $n-1$. This is a step toward inductively verifying Condition 5.

Now suppose that $\left\|v_{2}\right\|=n-1$. (This occurs if either $w^{\prime \prime}$ has length 3 or $w^{\prime \prime}$ has length 4 but is based at two vertices.) Then in $\Gamma(n)^{\prime}$ there is a copy of $\Gamma(v)$ attached to $v$ and a copy of $\Gamma\left(v_{2}\right)$ attached to $v_{2}$. In $\Gamma(v)$ there is exactly one $\Lambda_{\alpha}(v)$ which has a directed edge which starts at $v$ and has color $d_{2}$, and in $\Gamma\left(v_{2}\right)$ there is exactly one $\Lambda_{\beta}\left(v_{2}\right)$ which has a directed edge which starts at $v_{2}$ and has color $\left(d_{2}\right)^{-1}$. In the above we have identified the directed edge in $\Gamma(v)$ which starts at $v$ and has color $d_{2}$ with the directed edge $v v_{2}$ in $\Gamma(n)^{\prime}$ and we have identified the directed edge in $\Gamma\left(v_{2}\right)$ which starts at $v_{2}$ and has color $\left(d_{2}\right)^{-1}$ with the directed edge $v_{2} v$ in $\Gamma(n)^{\prime}$. In order that the resulting graph will be a prepermutation graph, we need to also identify the copies of $\Lambda_{\alpha}(v)$ and $\Lambda_{\beta}\left(v_{2}\right)$ in $\Gamma(n)^{\prime}$ so that the colors match up. This is possible by Lemma 1.iv). If $\left\|v_{1}\right\|=n-1$ (This occurs if either $\Lambda_{w^{\prime}}$ is a cycle of length $2 r+1$, based at one vertex of norm $n-r-1$, or if $A_{w^{\prime}}$ is a cycle of length $2 r$, based at two vertices of norm $n-r-2$.) then do the same thing with the appropriate cycles in $\Gamma(v)$ and $\Gamma\left(v_{1}\right)$. Continue this for all vertices of norm $n-1$ in $\Gamma(n)^{\prime}$, and call the resulting graph $\Gamma(n)$. By construction, $\Gamma(n)$ is a connected, prepermutation graph (this uses Lemma 1.v) if $\alpha$ and $\beta$ are 3-cycles), and all relators in $\Gamma(n)$ are a consequence of finite elements of $W$. Let $V(n)$ be the set of vertices in $\Gamma(n)$. Since $\Gamma(n-1)$ and each $\Gamma(v)$ are embedded in $\Gamma(n)$, we will identify them with their images in $\Gamma(n)$. In the case where we have identified $\Lambda_{\alpha}(v)$ with $\Lambda_{\beta}\left(v_{2}\right)$, we will think of their image as a single $\Lambda_{\omega}(v)$, even though it has more than one choice of labelled vertex.

We now investigate the possibility that some $A_{\omega}$ could be based at more than two vertices. Suppose $v, v_{1}$, and $v_{2}$ are as in Fig. 5.a or 5.b, with $\|v\|=\left\|v_{1}\right\|=\left\|v_{2}\right\|$ $=n-1$. If $\Lambda_{\alpha}(v)$ is identified with both $\Lambda_{\beta}\left(v_{2}\right)$ and $\Lambda_{\psi}\left(v_{1}\right)$, we find one of two possibilities (Fig. 8):
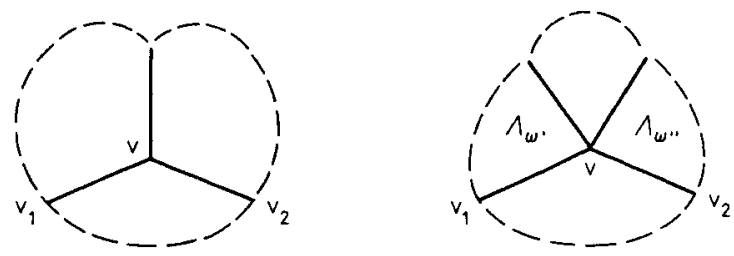

Fig. 8a, b

0

b

As usual, Fig. $8 \mathrm{a}$ is impossible. If Fig. $8 \mathrm{~b}$ arises, $D$ is 4-sided. Furthermore, if $v$ has minimal norm among all vertices producing $\Lambda_{\omega}$ 's based at three vertices, then $\Lambda_{w^{\prime}}$ and $A_{w^{\prime \prime}}$ have length 3 or 4 .

Since we will need to analyze the presentations coming from 4-sided fundamental domains, we list below the possibilities (Fig. 9).

Now, the situation where $A_{w}$ is based at three vertices actually does arise. Consider Fig. 9a with $p=q=3$. Then $G \cong\left\langle a, b \mid a^{3}, b^{3},(a b)^{r}\right\rangle$ is the $(3,3, r)$-triangle group from Fig. $2 b$. If $r=2, G$ is the tetrahedral group, so assume $r \geqq 3$. Figure 10 shows a piece of the group graph for the Euclidean case, $r=3$, with vertices labelled by their norms. The hyperbolic cases $(r>3)$ are similar. 
Edge Pairıngs

Relations

$a^{p} \cdot b^{q} \cdot|a b|^{r}$

a

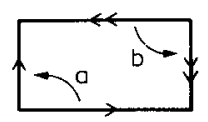

b

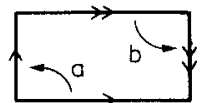

$a^{p} \cdot\left(a b^{2}\right)^{q}$

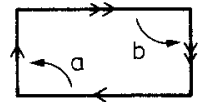

$(a b b a)^{p}$

$d$

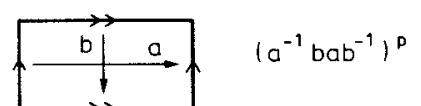

e

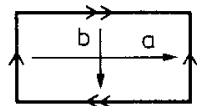

$\left(a^{-1} b a^{-1} b^{-1}\right)^{p}$

$(a b)^{p},\left(a b^{-1}\right)^{q}$
Local Cycle Structure
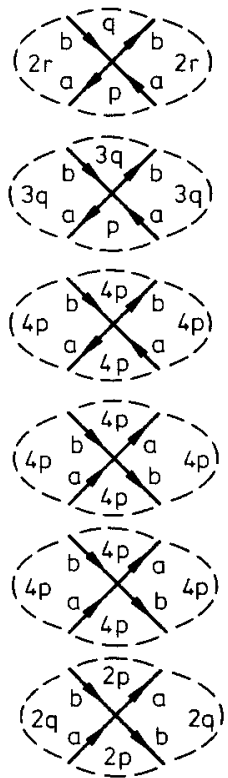

Remarks

p. $q \geqslant 3$

$r \geqslant 2$

$p \geqslant 3$

$q \geqslant 1$

$\left.l_{q}=1 \quad G \cong Z_{2 p}\right)$

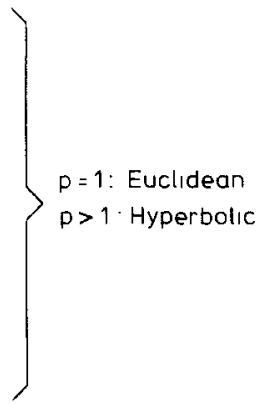

P. $q \geqslant 2$

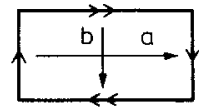

Fig. 9

Fig. 10

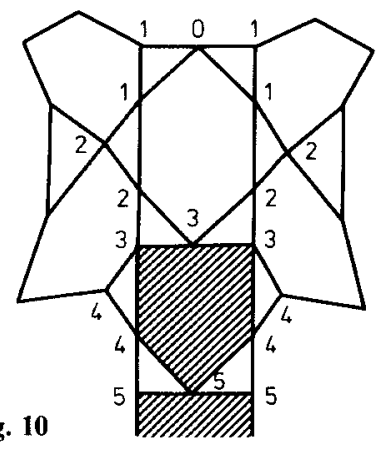

We claim these are the only such examples. For instance, suppose both $\Lambda_{w^{\prime}}$ and $\Lambda_{w^{\prime \prime}}$ (Fig. 8) have length 4 , and that $v, v_{1}, v_{2}$ have minimal norm among such triples. Working backwards, we find (Fig. 11):

Fig. 11

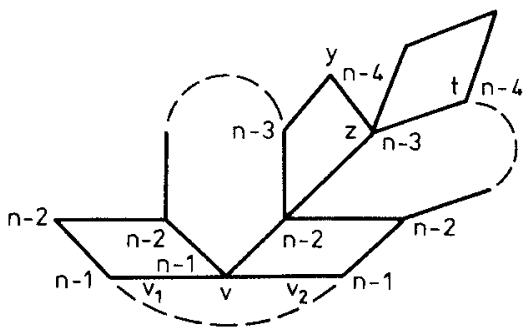


Fig. 12

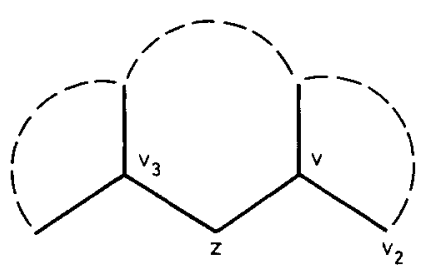

But the vertices $y, z, t$ violate Condition 4 for $\Gamma(n-3)$. A similar contradiction arises if one of $A_{w^{\prime}}, A_{w^{\prime \prime}}$ has length 3 and the other has length 4 . Refering to Fig. 9, we see that the only other possibility is Fig. $9 \mathrm{~b}$ with $q=1$, but this is a finite group.

Extend the norm on $V(n-1)$ to $V(n)$ by defining the norm of a vertex $v \in V(n)$ $-V(n-1)$ to be $\|v\|=\min \{\|v\|$ plus the edge-path distance in $\Gamma(v)$ from $v$ to $v$ : $v \in \Gamma(v)\}$.

It now remains to inductively verify Conditions $1-5$ for $\Gamma(n)$. Observe that Condition 1 is obvious, and we have already given the arguments necessary to verify Condition 5 (modulo checking that the $(3,3, r)$-triangle groups do not give rise to any other unusual behavior, but this is easy). The main work remaining consists in verifying Condition 4 . So let $z$ be a vertex of norm $n$. By a predecessor of $z$ we mean a vertex $v$, of norm $n-1$, connected by an edge to $z$. We need to show $z$ has at most two predecessors, as in Condition 4. Let $v$ be a predecessor of $z$. There are two main cases.

Case I: Every predecessor of $z$ has a unique predecessor.

Subcase A: $z$ is either $v_{1}$ or $v_{2}$ (Fig. 5a).

Suppose $z=v_{1}$. Of course, $z$ might have a predecessor belonging to $A_{w^{\prime}}$ (Fig. 12). If $z$ has another predecessor, it must have a predecessor that belongs to a cycle that shares either $v z$ or $v_{3} z$ with $A_{w^{\prime}}$. If it shares $v z$ it must also contain $v_{2} v$, and then the local picture makes $D 3$-sided. If it shares $v_{3} z$, then since $v_{3}$ has a unique predecessor, the situation is as shown in Fig. 12, and then the local picture at $v_{3}$ forces $D$ to be 3-sided.

Subcase B: $z \neq v_{1}, z \neq v_{2}$.

(1) If both $v_{1}$ and $v_{2}$ are predecessors of $z$, we have (Fig. 13):

Fig. 13

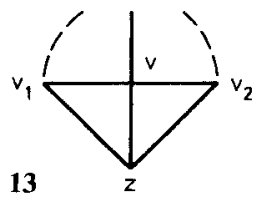

Hence, $D$ is 4-sided. But we see from Fig. 9 that the only infinite groups with 4sided Dirichlet regions, and 3-cycles in their presentations, are the $(3,3, r)$-triangle groups, in which case the two 3-cycles which emanate from a vertex are opposite, not adjacent. 
(2) If (say) $v_{2}$ is a predecessor of $z$, but $v_{1}$ is not, there are various possibilities. If a cycle through $v_{1}$ passes through $z$, we have (Fig. 14):

Fig. 14

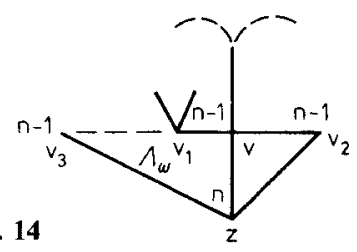

Since $A_{w} \subset \Gamma(n)-\Gamma(n-1)$, the vertices of $A_{w}$ have norm at least $n-1$. But if $\left\|v_{1}\right\|$ $=n, \Lambda_{w}$ violates Condition 5 . Thus, $\left\|v_{1}\right\|=n-1, A_{w}$ is a 4-cycle based at three vertices, and $G$ must be the tetrahedral group. It is easy to draw the entire group graph in this case, and indeed this situation does occur. On the other hand, if a cycle through $v_{2}$ passes through $z$, we find a picture exactly as in Fig. 14, but with $v$ replaced by $v_{2}$.

(3) If neither $v_{1}$ nor $v_{2}$ are predecessors of $z$, and a cycle through $v_{2}$, say, passes through $z$, we have (Fig. 15):

Fig. 15

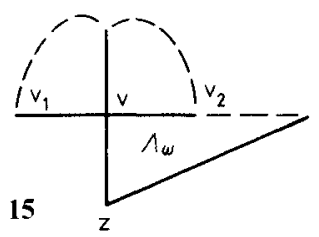

But Condition 5 forces $A_{w}$ to be a 4-cycle, based at three vertices $\left(\left\|v_{2}\right\|=n-1\right)$, so we have a $(3,3, r)$-triangle group. But this forces the cycles adjacent to $A_{w}$ to have length 3 , so $v_{1}$ is a predecessor of $z$ after all.

Case II: Some predecessor, $v$, of $z$ has two predecessors (Fig. 5b).

Subcase A: $\left\|v_{1}\right\|=\left\|v_{2}\right\|=n-1$.

(1) If both $v_{1}$ and $v_{2}$ are predecessors of $z, D$ is forced to have 5 sides.

(2) If neither $v_{1}$ nor $v_{2}$ are predecessors of $z$, then the existence of another predecessor gives rise to Fig. 16:

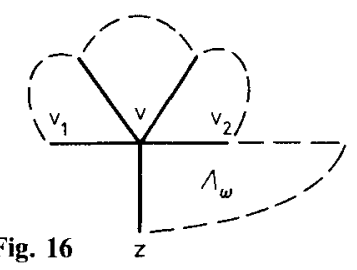

But then $A_{w}$ is based at three vertices, so $D$ has 4 sides, which contradicts the local picture at $v$.

(3) If $v_{1}$ is a predecessor of $z$, but $v_{2}$ is not, we must have Fig. 17: 
Fig. 17

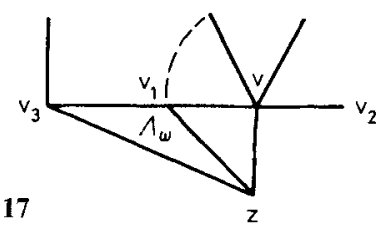

This is because a cycle containing $z$ and passing through $v_{2}$ would give a contradiction as in (2) above. If there was a vertex of $\Lambda_{w}$ in the interior of $v_{1} v_{3}$, Condition 5 would force $A_{w}$ to be based at three vertices, $A_{w}$ would have length 4 , and we would have the tetrahedral group. Thus, $A_{w}$ is a 3 -cycle, but now we have adjacent 3-cycles.

Subcase B: $\left\|v_{1}\right\|=n, v_{1} \neq z$.

We have already seen that no $\Lambda_{w}$ passing through $v_{1}$ can include $z$, and it is easy to see that at most one additional predecessor $\left(v_{2}\right)$ of $z$ can come via $v_{2}$.

Subcase C: $v_{1}=z$.

We begin with (Fig. 18):

Fig. 18

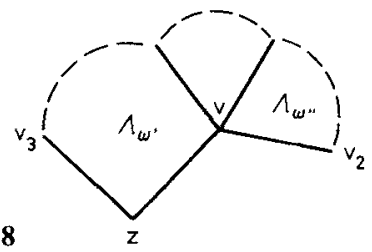

If $\left\|v_{3}\right\|=n$, a relatively short argument shows that $z$ has no predecessors coming via $v_{3}$. But previous arguments give that at most one predecessor $\left(v_{2}\right)$ comes via $v_{2}$. Thus, assume $\left\|v_{3}\right\|=n-1$, and $A_{w^{\prime}}$ has length 4 or 5 . If another predecessor comes via $v_{3}, G$ must be a $(3,3, r)$-triangle group, and the fact that $A_{w}$, has length 4 or 5 forces $G$ to be the tetrahedral group (but this does not arise). If $\left\|v_{2}\right\|=n$, we know (Condition 5) that no predecessor comes via $v_{2}$. Thus, assume $\left\|v_{2}\right\|=n-1$. Suppose a predecessor other than $v_{2}$ came via $v_{2}$. If $v_{2}$ is also a predecessor of $z$, we have a $(3,3, r)$-triangle group with two adjacent 3-cycles; whereas if $v_{2}$ is not a predecessor we again have a $(3,3, r)$-triangle group, but with two adjacent cycles of length greater than 3 . We conclude that the situation is (Fig. 19):

Fig. 19

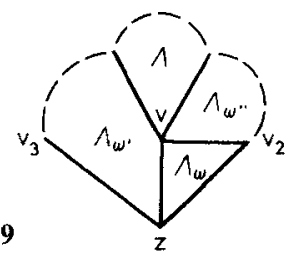

Now $\Lambda_{w^{\prime \prime}}$ must have length 4 , since otherwise there are adjacent 3 -cycles. But now this forces (Fig. 9a) $\Lambda_{w}$ to have length 4 , and we have a $(p, 3,2)$-triangle group, where $A$ has length $p$. Letting $z$ have minimal norm among all vertices with three predecessors, and working backwards, we find (Fig. 20): 


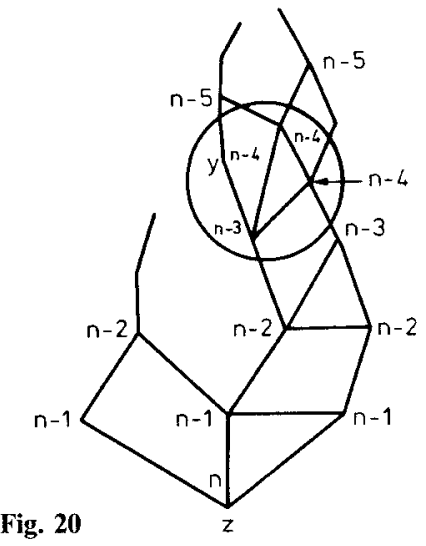

The circled portion contradicts minimality! However, our labelling implicity used that $p \geqq 6$, since otherwise $\|y\| \neq n-4$. In fact, this is precisely how vertices with three predecessors arise in the graphs of the octahedral $(p=4)$ and icosohedral $(p=5)$ groups. It is instructive to draw out these graphs - as vertices arise with more than two predecessors, the cycles begin "wrapping" around the graph. Eventually, a cycle wraps completely around the graph. The graph is then complete, and tessellates a sphere.

This completes the inductive verification of Condition 4 . We leave to the reader the relatively easy task of verifying Conditions 2 and 3. This now (finally!) completes the construction of $\Gamma$. As in [6], we find that $\Gamma=\bigcup_{n=0}^{\infty} \Gamma(n)$ is a connected prepermutation graph, each color emanates from every vertex of $\Gamma$, each finite word of $W$ is a relator at every vertex, and every relator of $\Gamma$ is a consequence of the finite words of $W$. Consequently, $\Gamma$ is the graph of the group with presentation $\left\langle\Sigma \mid\left(c_{1}\right)^{n_{1}}, \ldots,\left(c_{m}\right)^{n_{m}}\right\rangle$. In fact, $\Gamma$ is isomorphic to $K$ by an isomorphism $\varphi$ which takes $v_{0}$ to the base point $x$ in $K$ and is color-preserving. In addition, $\varphi$ is normpreserving between the norm \|\| on the vertices of $\Gamma$ and the norm $\|$ on $G$.

We now show that the $B(n)$ 's are balls. Recall that $G$ is a finitely generated, discrete, co-finite area group of hyperbolic or Euclidean isometries, $\Sigma$ is a geometric generating set for $G$ with respect to the Dirichlet region $D=D_{x}$, $\Sigma$ has no elements of order two, and for each integer $n \geqq 0 B(n)=\operatorname{closure}(\cup\{g D:|g| \leqq n\})$.

Lemma 2. For every integer $n \geqq 0, B(n)$ is a ball.

Proof. First exclude the $(3,3, r)$-triangle groups with 4-sided Dirichlet regions. The proof is by induction on $n$. The lemma is valid for $n=0$ since $B(0)=\operatorname{cl}(D)$ is a ball. Let $n>0$, and assume that $B(n-1)$ is a ball. Let $\left\{b_{1}, \ldots, b_{k}\right\}$ be an enumeration of the elements in $G$ of norm $n$. For each $i \in\{1, \ldots, k\}$, let $C_{i}=B(n-1) \cup c l\left(b_{1} D\right.$ $\left.\cup \ldots \cup b_{i} D\right)$. Let $C_{0}=B(n-1)$. Then $B(n)=C_{k}$, so it suffices to show that each $C_{i}$ is a ball.

Let $i \in\{1, \ldots, k\}$, and assume by induction that $C_{i-1}$ is a ball. We will show that $C_{i}$ is a ball by showing that $C_{i-1} \cap c l\left(b_{i} D\right)$ is a closed interval. By Condition $4, b_{i} D$ intersects $B(n-1)$ in either one edge or in two edges which have a vertex in 
common. By Condition 5 , if $b_{i} D \cap b_{j} D$ is not empty for some $j \neq i$, then $b_{i} D \cap b_{j} D$ is an interval and $b_{i} D \cap b_{j} D \cap B(n-1)$ is a point in the boundary of $B(n-1)$. Thus $C_{i-1} \cap b_{j} D$ is an interval and hence $C_{i}$ is a ball.

For the $(3,3, r)$-triangle groups, it is easy to see that each $C_{i}$ is a ball if one is careful in enumerating the elements of norm $n$.

Note that the proof of Lemma 2 actually shows that, except for the $(3,3, r)$ triangle groups, if $B(n)$ is built from $B(n-1)$ by adding the domains of norm $n$, one at a time, in any order, the result is a ball at each stage.

Actually, Brenner and Lyndon [4] have shown the following remarkable fact. Let $\Pi$ be any cellulation of the plane (i.e. $\Pi$ is a union of polygons covering the plane, and two polygons are either disjoint, meet in a vertex, or meet along an edge). Pick a base polygon $P_{0} \in \Pi$. Define $P \in \Pi$ to have length 1 if $P \cap P_{0}$ is an edge. Assume inductively that polygons of length $n-1$ have been defined, let $B(n-1)$ be the union of all $P \in \Pi$ of length $\leqq n-1$, and define $P \in \Pi$ to have length $n$ if $P \cap B(n-1)$ is a union of edges. Then, assuming that every $P \in \Pi$ has at least six sides, each $B(n)$ is a topological ball, and in fact, if we build $B(n)$ from $B(n-1)$ by adding length $n$ polygons in any order, the result is a ball at each stage.

Brenner and Lyndon made no assumptions that a group acts transitively on the polygons, which is the case for us. Our approach, however, allows us to find a "recursive structure" on the elements of $G$, thereby showing the rationality of the growth function (see below). Note that the proof of Lemma 2 also establishes the following result.

Lemma 3. Let $n \geqq 0$, and $g, h \in G$ with $|g|=|h|=n+1$. Then $B(n) \cap g D$ is an interval, and $g D \cap h D$ is either empty or an interval which intersects $B(n)$ in a point (again excluding the $(3,3, r)$-triangle groups $)$.

Let $g(z)$ be the growth series of $G$ with respect to the generating set $\Sigma$. We know from [5] that $g$ is the power series of a rational function $f$. Cannon proves this by giving a finite labelling of $G$ by "cone-types", and using this to show that the coefficients of $g$ satisfy a linear recursion. Using the fact that $\Sigma$ is a geometric generating set, we will describe another finite labelling of $G$ (analogous to Cannon's method in [6]) that shows that the coefficients of $g$ satisfy a linear recursion. This gives an alternate proof in this case that $g$ is the power series of a rational function, and makes the proof of our main theorem much easier.

For each $\Lambda_{\omega}(v)$ in $\Gamma$, denote by $\Lambda_{\omega}(\varphi(v))$ the image $\varphi\left(\Lambda_{\omega}(v)\right)$, as a subgraph of $K$ which is colored by $\Sigma$ and has a base vertex labelled by $\varphi(v)$. We will partition the elements of $G$ into finitely many types, as follows. The identity element of $G$ is in a type by itself, and each element $g \in \Sigma$ (these are the elements in $G$ of norm one) is the only element in its type. Let $g \in G$ with $|g| \geqq 2$. Then $g x$ is joined by either one or two edges in $K$ to vertices of norm $|g|-1$. Each of these edges is contained in exactly two $\Lambda_{\omega}$ 's in $K$. Let $A(g)$ denote the union of these two or three $\Lambda_{\omega}$ 's, as a colored subgraph of $K$ with certain vertices labelled as base vertices. (Each $A_{\omega}$ has one, two, or three choices of labelled vertex, and we will call each such choice a base vertex. However, we will forget the particular labelling of each base vertex.) Given two elements $g, g^{\prime} \in G$ with $|g| \geqq 2$ and $\left|g^{\prime}\right| \geqq 2$, then $g$ and $g^{\prime}$ are in the same type if and only if there is a color-preserving isomorphism $\tau: A(g) \rightarrow \Lambda\left(g^{\prime}\right)$ such that $\tau$ takes the 
base vertices of $\Lambda(g)$ to the base vertices of $\Lambda\left(g^{\prime}\right)$ and $\tau(g x)=g^{\prime} x$. Let $t(g)$ denote the type of $g$, and let $\left\{t_{1}, \ldots, t_{r}\right\}$ be an enumeration of the non-identity types.

Let $j \in\{1, \ldots, r\}$, and choose an element $g \in G$ with $t(g)=t_{j}$. We define $n(j)(=1$ or 2) to be the cardinality of $\{h \in G:|h|=|g|-1$ and $g x$ and $h x$ are connected by an edge in $K\}$, and for each $i \in\{1, \ldots, r\}$ we define $m(i, j)$ to be the cardinality of $\{h \in G$ : $|h|=|g|+1, g x$ and $h x$ are connected by an edge in $K$, and $\left.t(h)=t_{i}\right\}$. Then $n(j)$ is the number of predecessors of $g$, and $m(i, j)$ is the number of elements of type $t_{i}$ which have $g$ as a predecessor.

We claim that $n(j)$ and $m(i, j)$ depend only on $i$ and $j$ and not on the representative element $g$. This is clear if $|g|=1$, since in this case $g$ is the only element with type $t_{j}$. So assume that $|g| \geqq 2$. Then $n(j)=1$ if $g x$ is in the boundary of exactly three edges in $\Lambda(g)$ (see Fig. 5), and $n(j)=2$ if $g x$ is in the boundary of four edges in $A(g)$. In particular, $n(j)$ depends only on the type of $g$. Let $N(g)=\{h x$ : $h \in G,|h|=|g|+1$, and there is an edge in $K$ joining $g x$ and $h x\}(N(g)$ is the next generation of $g$ ). Then $m(i, j)$ is the cardinality of $\left\{h \in N(g): t(h)=t_{i}\right\}$, so we need to show that the distribution by types of the elements of $N(g)$ depends only on the type of $g$. Let $\Lambda^{\prime}(g)$ be the union of $\Lambda(g)$ and all edges in $K$ whose boundaries contain $g x$ (see Fig. 21). Since $\Lambda^{\prime}(g)$ has exactly one edge of each color emanating from $g x, \Lambda^{\prime}(g)$ is determined up to color-preserving isomorphism by the type of $g$. Let $h \in N(g)$, and let $e$ be the edge in $A^{\prime}(g)$ joining $g x$ and $h x$. Then $e$ is contained in two $\Lambda_{\omega}$ 's, $\Lambda_{w}$ and $\Lambda_{w^{\prime}}$, in $\Lambda(h)$, and by Lemma 1.iv) these $\Lambda_{\omega}$ 's are determined by the color of $e$. If the union of these two $A_{\omega}$ 's is $A(h)$, then the $A_{\omega}$ 's in $A(h)$ are determined by the color of $e$. Suppose $A(h) \neq \Lambda_{w} \cup \Lambda_{w^{\prime}}$, and $h x$ is not in $A(g)$. Then $h$ has a second predecessor, and, unless $G$ is a $(3,3, r)$-triangle group, we can assume without loss of generality that it is $g_{2}$, so we have Fig. 22 .
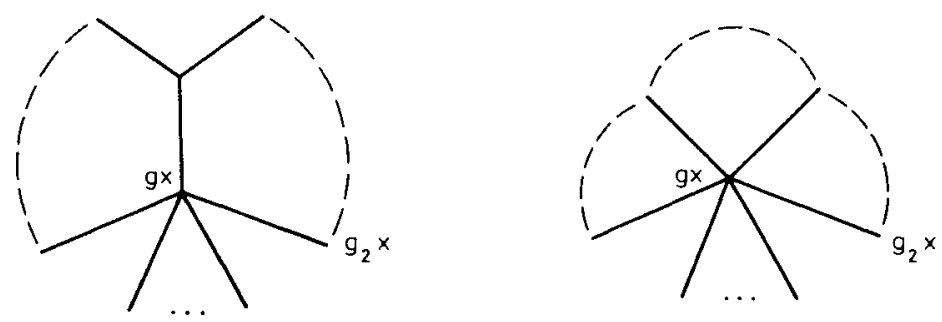

Fig. 21a, b a

b
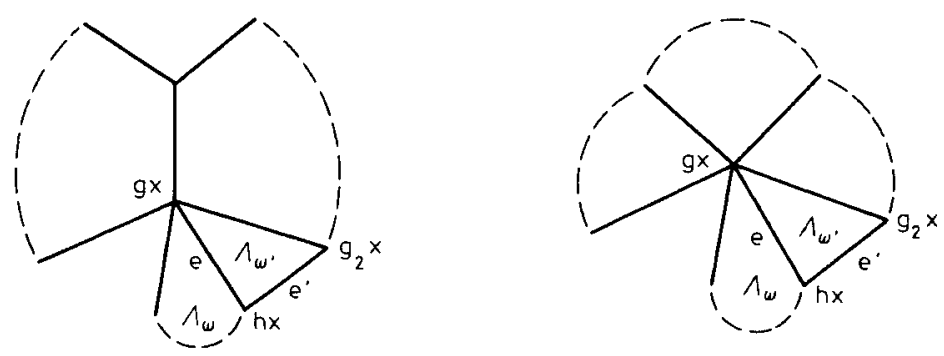

Fig. 22a, b a 
Fig. 23a, b a
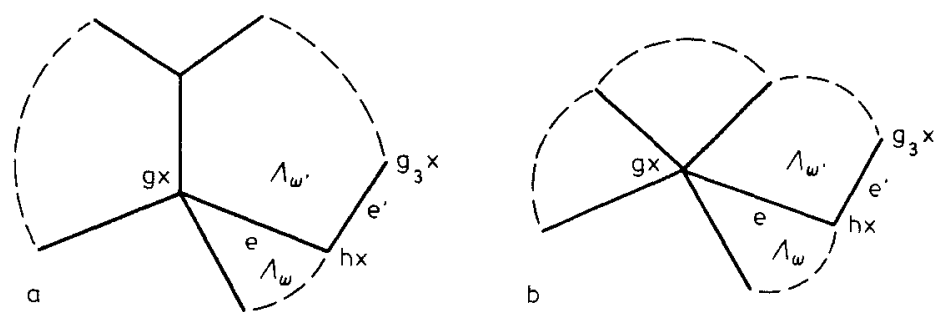

This occurs when $A_{w^{\prime}}$, has length 3 and $\left|g_{2}\right|=|g|$. The third $\Lambda_{\omega}$ of $A(h)$ is the other $A_{\omega}$ attached to the edge $e^{\prime}$. Since the color of $e^{\prime}$, the length of $A_{w^{\prime}}$, and $\left|g_{2}\right|$ are determined by the type of $g$, in this case the $A_{\sigma}$ ' ' $\sin A(h)$ are determined by the color of $e$ and the type of $g$.

The final case is that $\Lambda(h) \neq \Lambda_{w} \cup \Lambda_{w^{\prime}}$ and $h x \in \Lambda(g)$. Without loss, we can assume that $h=g_{2}$, so we have Fig. 23. This occurs when $\left|g_{3}\right|=|g|$, and the third $A_{\omega}$ in $A(h)$ is determined by the color of $e^{\prime}$. Since this information is determined by the type of $g$, in this case also the $A_{\omega}$ 's in $A(h)$ are determined by the color of $e$ and the type of $g$.

It remains to show that the base vertices of $\Lambda(h)$ are determined by the color of $e$ and the type of $g$. It is easy to show that the base vertices of $A(h)$ are the obvious ones if $G$ is not a $(3,3, r)$-triangle group or a $(3,4, r)$-triangle group. We leave it to the reader to check that the type of $g$ determines the base vertices of $\Lambda(h)$ for the $(3,3, r)$-and $(3,4, r)$-triangle groups. Since the type of $g$ determines the colors of the edges in $\Lambda^{\prime}(g)$, each $m(i, j)$ depends only on the type $t_{j}$ and not on the representative $g$ of that type.

Let $\mathbf{A}$ be the $r \times r$ matrix with $\mathbf{A}_{i j}=m(i, j) / n(i)$, and let $\mathbf{v}$ be the $r \times 1$ matrix whose $i^{\text {th }}$ entry is the cardinality of $\left\{g \in G:|g|=1\right.$ and $\left.t(g)=t_{i}\right\}$. Then for each positive integer $n$, the matrix $\mathbf{A}^{n} \mathbf{v}$ has $i^{\text {th }}$ entry the cardinality of $\{g \in G:|g|=n+1$ and $\left.t(g)=t_{i}\right\}$. Let $(1 \ldots 1)$ be the $1 \times r$ matrix with all entries 1 . Then the $n^{\text {th }}$ coefficient $a_{n}$ of $g$ is $(1 \ldots 1) \mathbf{A}^{n-1} \mathbf{v}$.

Lemma 4. With the notation as above, $g$ is the Maclaurin series of the rational function $f(z)=1+(1 \ldots 1)(\mathbf{I}-\mathbf{z A})^{-1} z \mathbf{v}$.

Proof. By the above,

$$
\begin{aligned}
g(z) & =1+a_{1} z+\ldots+a_{n} z^{n}+\ldots \\
& =1+(1 \ldots 1) z \mathbf{v}+\ldots+(1 \ldots 1) \mathbf{A}^{n-1} z^{n} \mathbf{v}+\ldots \\
& =1+(1 \ldots 1)\left(\mathbf{I}+z \mathbf{A}+\ldots+z^{n-1} \mathbf{A}^{n-1}+\ldots\right) z \mathbf{v} .
\end{aligned}
$$

But this is the Maclaurin series of the rational function

$$
f(z)=1+(1 \ldots 1)(\mathbf{I}-z \mathbf{A})^{-1} z \mathbf{v} .
$$

In computing examples, it is often possible to exploit symmetries in the Dirichlet region in order to reduce the number of types. For convenience, we will give a definition of the kind of structure we need. Let $G$ be a finitely generated group, and $\Sigma$ a finite generating set for $G$. A recursive structure for $(G, \Sigma)$ is a 
partition of the elements of $G$-\{identity $\}$ into finitely many equivalence classes $\left\{t_{1}, \ldots, t_{s}\right\}$, called types, so that for each $i, j \in\{1, \ldots, s\}$ and $g \in G$ with type $t_{j}$, the cardinality, $n(j)$, of $\left\{h \in G:|h|_{\Sigma}=|g|_{\Sigma}-1\right.$ and $g$ and $h$ are connected by an edge in the graph of $G$ with respect to $\Sigma\}$ and the cardinality, $m(i, j)$, of $\left\{h \in G:|h|_{\Sigma}=|g|_{\Sigma}+1\right.$, the type of $h$ is $t_{i}$, and $g$ and $h$ are connected by an edge in the graph of $G$ with respect to $\Sigma\}$ depend only on the type of $g$. The recursion matrix is the $s \times s$ matrix $\mathbf{A}$ with $\mathbf{A}_{i j}$ $=m(i, j) / n(i)$ and the initial vector is the $s \times 1$ matrix $\mathbf{v}$ with $i^{\text {th }}$ entry the cardinality of $\left\{g \in G:|g|_{\Sigma}=1\right.$ and the type of $g$ is $\left.t_{i}\right\}$. Note that a recursive structure is all that was used to prove Lemma 4.

\section{§2. The Euler characteristic}

Let $G$ be a finitely generated, discrete group of isometries of $\mathbb{H}^{2}$ such that $\mathbb{H}^{2} / G$ has finite area, and $\Sigma$ a finite generating set for $G$ which is geometric with respect to the Dirichlet region $D=D_{x}$ and has no elements of order two. Let $\left\{t_{1}, \ldots, t_{r}\right\}$ be a recursive structure for $(G, \Sigma)$, and for each $g \in G$-\{identity $\}$ let $t(g)$ denote the type of $g$. Let $g \in G$-\{identity , and let $n=|g|$. Let $\sigma(g)$ be the number of edges of $g\left(D_{x}\right)$ $\cap B(n-1)$, and let $\Theta(g)$ be the sum, over the vertices $v$ in $g\left(D_{x}\right) \cap B(n-1)$, of the angle in $B(n-1)$ at the vertex $v$ divided by the cardinality of $\{h \in G:|h|=n$ and $v \in h(D)\}$. So $\sigma(g)$ counts the number of sides with which $g(D)$ is attached to $B(n-1)$ and $\Theta(g)$ counts the portion of the angle sum of $\partial B(n-1)$ corresponding to $g\left(D_{x}\right) \cap B(n-1)$. The recursive structure is geometric if for each $g \in G$-\{identity\}, $\sigma(g)$ and $\Theta(g)$ depend only on the type of $g$. Note that the recursive structure defined in $\S 2$ is clearly geometric. If the recursive structure is geometric, then the attaching vector is the $1 \times r$ matrix $w$ whose $j^{\text {th }}$ entry is $(\Theta(g) / \pi)-\sigma(g)$, for any $g \in G$ with type $t_{j}$.

Theorem 1. Let $G$ be a discrete group of isometries of $\mathbb{H}^{2}$ with $\mathbb{H}^{2} / G$ of finite area, $\Sigma$ a geometric generating set for $G$ with no elements of order two, and $\mathbf{A}, \mathbf{v}, \mathbf{w}$ determined as above by a geometric recursive structure for $(G, \Sigma)$. Then the growth series for $G$ with respect to $\Sigma$ is the Maclaurin series of the rational function

$$
f(z)=1 / \chi+(1-z)(2 \chi)^{-1} \mathbf{w}(\mathbf{I}-z \mathbf{A})^{-1} \mathbf{v},
$$

where $\chi$ is the rational Euler characteristic of $G$ (the orbifold Euler characteristic of $\left.\mathbb{H}^{2} / G\right)$.

Proof. At this point, it is somewhat more natural to work not with the growth series $g(z)$, but with the cumulative growth series $h(z)=\sum_{n=0}^{\infty} b_{n} z^{n}$, where $b_{n}=\left(1+a_{1}\right.$ $\left.+\ldots+a_{n}\right)$ is the number of elements of $G$ with length $\leqq n$. These are clearly related by $(1-z) h(z)=g(z)$.

For every $n \geqq 0$, let $\sigma(n)$ be the number of sides of $B(n)$ and let $\Theta(n)$ be the sum of the angles at the vertices of $B(n)$. Since $\mathbf{A}^{n} \mathbf{v}$ is the distribution by types of the elements of length $n+1, \mathbf{w A}^{n} \mathbf{v}=(\Theta(n) / \pi)-\sigma(n)$. By the Gauss-Bonnet theorem and Lemma 2, the area of $B(n)=\pi(\sigma(n)-2)-\Theta(n)=-\pi\left(2+\mathbf{w A}^{n} \mathbf{v}\right)$. But also by GaussBonnet, we have that the area of $D=\operatorname{area}\left(\mathbb{H}^{2} / G\right)=-2 \pi \chi$, hence the area of $B(n)$ 
$=-2 \pi \chi b_{n}$, so that $b_{n}=1 / \chi+(1 / 2 \chi) \mathbf{w} \mathbf{A}^{n} \mathbf{v}$. Thus,

$$
\begin{aligned}
h(z) & =\sum_{n=0}^{\infty}\left(1 / \chi+(1 / 2 \chi) \mathbf{w} \mathbf{A}^{n} \mathbf{v}\right) z^{n} \\
& =1 /(1-z) \chi+(1 / 2 \chi) \mathbf{w}(\mathbf{I}-z \mathbf{A})^{-1} \mathbf{v} .
\end{aligned}
$$

Multiplying by $1-z$ now proves the theorem.

An immediate consequence of Theorem 1 is that $f(1)=1 / \chi$ if $\mathbf{I}-\mathbf{A}$ is invertible. Since 1 is not an eigenvalue of $A$ for a generic square matrix $A$, one might expect $f(1)=1 / \chi$ for most geometric generating sets for Fuchsian groups. As we will see in the next section, there are geometric generating sets for which $\mathbf{I}-\mathbf{A}$ is not invertible and $f(1) \neq 1 / \chi(G)$.

\section{§3. Examples}

We want to describe some examples and computations of growth functions for Fuchsian groups with geometric generators $\Sigma$. We first consider growth functions associated to tessellations of $\mathbb{H}^{2}\left(\mathbb{E}^{2}, S^{2}\right)$ by regular $n$-gons, $n$ even, $n \geqq 4$, with vertex angle $2 \pi / p, p \geqq 3$. It is well-known that such regular polygons exist. Except for $n=4, p=3$ (spherical quadrilateral), $n=4, p=4$ (Euclidean square), and $n=6$, $p=3$ (Euclidean hexagon), they are hyperbolic. We will refer to them as $(n, 2 \pi / p)$ gons.

Suppose an $(n, 2 \pi / p)$-gon is the fundamental domain for a (torsion-free, closed) surface group. An Euler characteristic argument shows that if the surface is orientable, genus $g$, then $g=\frac{1}{2}\left(1+\frac{n}{2}-\frac{n}{p}\right)$, so that $p \mid n$ and $\frac{n}{2}+\frac{n}{p}$ is odd. If the surface is non-orientable, genus $g$, then $g=1+\frac{n}{2}-\frac{n}{p}$, hence $p \mid n$.

Conversely, if $p \mid n$, an $(n, 2 \pi / p)$-gon is a fundamental domain for a surface group. This is seen in the following way. If $g=\frac{1}{2}\left(1+\frac{n}{2}-\frac{n}{p}\right)$ is an integer, let $F_{g}$ be a closed, orientable surface of genus $g$. Now $F_{g}$ has a spine with $2 g$ edges and one vertex of valence $4 g$. Perform Whitehead moves to create valence $p$ vertices, one at a time, from the original vertex. When this has been done $\left(\frac{n}{p}-1\right)$ times, we have a new spine with $\frac{n}{p}$ vertices, each of valence $p$. Equivalently, there are edge pairings on a polygon with $n=2 p(2 g-1) /(p-2)$ sides so that each vertex cycle has length $p$. (If $\frac{1}{2}\left(1+\frac{n}{2}-\frac{n}{p}\right)$ is not an integer, one can do the same construction on a nonorientable surface of genus $g=1+\frac{n}{2}-\frac{n}{p}$. $)$ Now let $D$ be an $(n, 2 \pi / p)$-gon in $\mathbb{H}^{2}$ (except for $(4, \pi / 2)$ and $(6,2 \pi / 3)$, which give tessellations of $\left.\mathbb{E}^{2}\right)$. Since $D$ is regular, we can pick hyperbolic isometries to give the desired edge pairings, and now Poincarés Theorem for Fundamental Polygons $[19,14]$ shows that the edge pairings generate a discrete, torsion-free group having $D$ as a fundamental domain. 
For fixed $n$, this gives only a finite collection of $(n, 2 \pi / p)$-gons. We can do much better if we allow torsion in $G$. For example, suppose $(p, n) \geqq 3$, and pick $q$ so that $q|p, q| n, q \geqq 3$. Then there is an $(n, 2 \pi / q)$-gon which is the fundamental domain for a torsion-free group. Replace the $(n, 2 \pi / q)$-gon by $D=(n, 2 \pi / p)$-gon, keeping the same edge pairings. The angle sum for each vertex cycle is now $2 \pi /(p / q)$, so Poincare's Theorem again shows that the edge pairings generate a discrete group with $D$ as fundamental domain.

Of course, this construction has a lot of symmetry - every vertex has branching index $p / q$. When $(p, n) \leqq 2$, one could imagine that a less symmetric construction would produce an $(n, 2 \pi / p)$-gon as fundamental domain. For example, let $n=4$. If $4 \mid p$, the edge pairings in Fig. 9c, d, or e, give a Fuchsian group $G(p>4)$, and $\mathbb{H}^{2} / G$ is a torus or Klein bottle with one vertex, branching index $p / 4$. If 4 does not divide $p$ but $2 \mid$ p, then Fig. 9 a gives a $(p, p, p / 2)$-triangle group. In the quotient $S^{2}$, two vertices have branching index $p$, one vertex has branching index $p / 2$. If $3 \mid p$, then Fig. $9 \mathrm{~b}$ gives a discrete group with quotient the projective plane, one vertex having index $p$, the other having index $p / 3$. (If $p=3$, the group is $\mathbb{Z}_{6}-S^{2}$ is tiled by six $(4,2 \pi / 3)$-gons - but otherwise the group is Fuchsian.) But if $p$ is odd and 3 does not divide $p$, the $(4,2 \pi / p)$-gon is not a fundamental domain for any discrete group (even allowing reflections).

Consider the general problem of tiling $\mathbb{H}^{2}$ by $(n, 2 \pi / p)$-gons. If $p$ is even, and we allow reflections, we simply use the Coxeter group based on the polygon $\left\langle a_{1}, \ldots, a_{n} \mid a_{i}^{2},\left(a_{i} a_{i+1}\right)^{p / 2}\right\rangle$. The case $(p, n)=1$ is clearly more complicated combinatorially, as the example above shows, and we do not know the exact conditions on $p$ and $n$ which allow the construction of a discrete group having the $(n, 2 \pi / p)$-gon as a fundamental domain. Nevertheless, such tilings always exist. In fact, one can view the discussion which follows as a construction of the tilings. The type analysis shows how to inductively build the combinatorial ball $B(n)$, where length is taken in the sense of Brenner-Lyndon, as described earlier. It will be clear that each $B(n)$ has a non-singular, complete hyperbolic structure in its interior, and its boundary is a broken geodesic. Since each $B(n)$ is a topological ball, $\bigcup_{n=0}^{\infty} B(n)$ is a simply connected, complete, hyperbolic manifold, hence $\mathbb{H}^{2}$.

Thus, in what follows, we will not worry about the existence of a group $G$ having the $(n, 2 \pi / p)$-gon as a fundamental domain. The discussion is written as though $G$ exists - if $G$ does not exist, think of $G$ as the set of polygons in the tiling, interpret length in the sense of Brenner-Lyndon, and let $\chi(G)=(-1 / 2 \pi)$ area $(D)$.

Given $n(n \geqq 4)$ and $p(p \geqq 3)$, we will find a geometric recursive structure for $(G, \Sigma)$, where $G$ is the discrete group generated by the edge pairing $\Sigma$ on an $(n, 2 \pi / p)$-gon, and explicitly calculate the initial vector $\mathbf{v}$, recursion matrix $\mathbf{A}$, and growth function $f$. We will see that the growth function depends only on $n$ and $p$, and not on the choice of edge pairings. The argument is divided into the following four cases: (1) $p=3$, (2) $p=4$, (3) $p=2 k, k \in \mathbb{Z}, k>2$, and (4) $p=2 k+1, k \in \mathbb{Z}, k \geqq 2$.

Let $p=3$. If $n=4(n=5)$, then the growth function is $1+4 z+z^{2}(1+5 z$ $\left.+5 z^{2}+z^{3}\right)$. So assume $n \geqq 6$. Our recursive structure will have two types, $t_{1}$ and $t_{2}$. Let $g \in G$ with $|g|_{\Sigma}=r \geqq 1$. The type of $g$ is $t_{1}$ if $g(D) \cap B(r-1)$ is a single edge $e$ and both vertices of $\partial e$ have angle $2 \pi / 3$ in $B(r-1)$. The type of $g$ is $t_{2}$ if $g(D)$ $\cap B(r-1)$ consists of two edges with a vertex in common, the interior vertex of $g D$ 


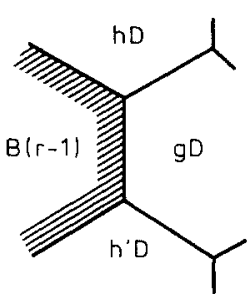

Fig. 24a, b

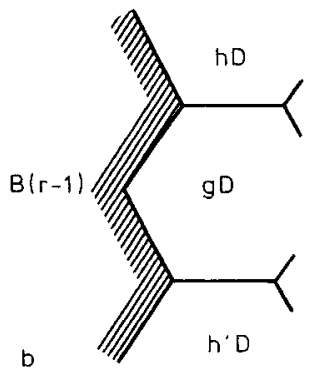

$\cap B(r-1)$ has angle $4 \pi / 3$ in $B(r-1)$, and the other two vertices each have angle $2 \pi / 3$ in $B(r-1)$. So $n(1)=1$ and $n(2)=2$. We need to show that every element in $G$-\{identity $\}$ has one of these two types, and that this does give a recursive structure for $(G, \Sigma)$. Suppose $r \geqq 1$, and $g \in G$ with $|g|=r$ and $t(g)=t_{1}$. By Lemmas 2 and 3 , there exist $h, h^{\prime} \in G$ so that $|h|=\left|h^{\prime}\right|=r$ and the local picture near $g D$ in $B(r)$ is that of Fig. 24a. Thus $g x$ is connected by edges in $K$ to $n-5$ vertices of type $t_{1}$ and to two vertices of type $t_{2}$. Similarly, if $|g|=r$ and $t(g)=t_{2}$, then there are elements $h, h^{\prime}$ in $G$ so that the local picture near $g D$ in $B(r)$ is that of Fig. $24 \mathrm{~b}$. In this case $g x$ is connected by edges in $K$ to $n-6$ vertices of type $t_{1}$ and to two vertices of type $t_{2}$. Since every element of norm one has type $t_{1}$, this shows inductively that every element of $g$ has type $t_{1}$ or type $t_{2}$ and that $\left\{t_{1}, t_{2}\right\}$ gives a recursive structure with initial vector $\mathrm{v}=\left[\begin{array}{l}n \\ 0\end{array}\right]$ and recursion matrix $\mathbf{A}=\left[\begin{array}{cc}n-5 & n-6 \\ 1 & 1\end{array}\right]$. By Lemma 4, the growth series $g$ for $G$ with respect to $\Sigma$ is the Maclaurin series of the rational function $f(z)=\frac{z^{2}+4 z+1}{z^{2}+(4-n) z+1}$. Notice that $n=6$ gives the hexagonal tiling of $\mathbb{E}^{2}$, and $f$ has a double pole at $z=1$.

Now let $p=4$. An element $g \in G$ with $|g|=r \geqq 1$ has type $t_{1}$ if $g D \cap B(r-1)$ is a single edge with both vertices of angle $\pi / 2$ in $B(r-1)$, and $g$ has type $t_{2}$ if $g D$ $\cap B(r-1)$ is two edges with a vertex in common, the two exterior vertices of $g D$ $\cap B(r-1)$ have angle $\pi / 2$ in $B(r-1)$, and the interior vertex has angle $3 \pi / 2$ in $B(r-1)$. An argument similar to the above shows that $\left\{t_{1}, t_{2}\right\}$ gives a recursive structure for $(G, \Sigma)$ with initial vector $\mathbf{v}=\left[\begin{array}{l}n \\ 0\end{array}\right]$ and recursion matrix $\mathbf{A}=\left[\begin{array}{cc}n-3 & n-4 \\ 1 & 1\end{array}\right]$. So the growth function $f(z)=\frac{z^{2}+2 z+1}{z^{2}+(2-n) z+1}$. Notice that $n=4$ gives the tiling of $\mathbb{E}^{2}$ by squares, with the "usual" growth function $(1+z)^{2} /(1-z)^{2}$ for $\mathbb{Z}^{2}$.

Now suppose $p=2 k$. In this case there are $k$ types. For $i \in\{1, \ldots, k-1\}$, an element $g \in G$ with $|g|=r \geqq 1$ is of type $t_{i}$ if $g D \cap B(r-1)$ is a single edge $e$, one vertex of $\partial e$ has angle $\pi / k$ in $B(r-1)$, and the other vertex has angle $(2 i-1) \pi / k$ in $B(r-1)$. An element $g \in G$ is of type $t_{k}$ if $g D \cap B(r-1)$ consists of two edges with a vertex in common, the exterior vertices of $g D \cap B(r-1)$ each have angle $\pi / k$ in $B(r-1)$, and the interior vertex has angle $(2 k-1) \pi / k$ in $B(r-1)$. Suppose $i \in\{1, \ldots, k-1\}$, and 


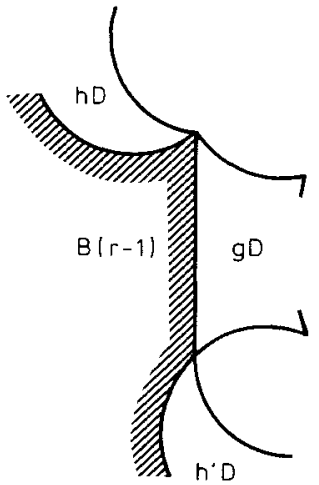

Fig. 25a, $b$

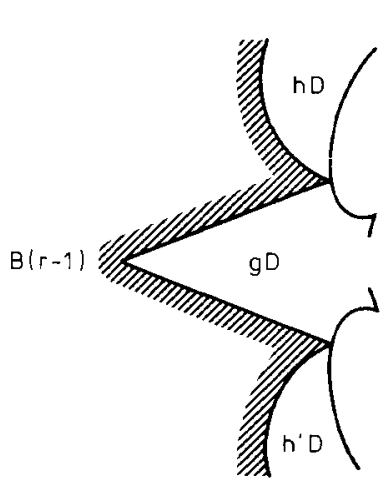

b

$g \in G$ with $t(g)=t_{i}$ and $|g|=r$. By Lemmas 2 and 3, there exist $h, h^{\prime} \in G$ so that $|h|$ $=\left|h^{\prime}\right|=r$ and the local picture in $B(r)$ near $g D$ is that of Fig. 25a. Thus $g x$ is connected by edges in $K$ to $n-1$ vertices of norm $r+1$, of which $n-3$ have type $t_{1}$, one has type $t_{2}$, and one has type $t_{i+1}$. If $g$ has type $t_{k}$, then the local picture near $g D$ in $B(r)$ is that of Fig. 25b. In this case $g x$ is connected by edges in $K$ to $n-2$ vertices of norm $r+1$, of which $n-4$ have type $t_{1}$ and two have type $t_{2}$. Since every element of norm one has type $t_{1}$, this shows that each element of $g \in G$-\{identity\} has type in $\left\{t_{1}, \ldots, t_{k}\right\}$. Hence $\left\{t_{1}, \ldots, t_{k}\right\}$ gives a recursive structure with initial vector

$$
\mathbf{v}=\left[\begin{array}{c}
n \\
0 \\
0 \\
0 \\
\vdots \\
0 \\
0
\end{array}\right]
$$

and recursion matrix

$$
\mathbf{A}=\left[\begin{array}{cccccccc}
n-3 & n-3 & n-3 & n-3 & \ldots & n-3 & n-3 & n-4 \\
2 & 1 & 1 & 1 & \ldots & 1 & 1 & 2 \\
0 & 1 & 0 & 0 & \ldots & 0 & 0 & 0 \\
0 & 0 & 1 & 0 & \ldots & 0 & 0 & 0 \\
\vdots & \vdots & & & . & & \vdots & \vdots \\
0 & 0 & 0 & 0 & \ldots & 1 & 0 & 0 \\
0 & 0 & 0 & 0 & \ldots & 0 & 1 / 2 & 0
\end{array}\right],
$$

and the growth function for $G$ with respect to $\Sigma$ is

$$
f(z)=\frac{z^{k}+2 z^{k-1}+2 z^{k-2}+\ldots+2 z^{2}+2 z+1}{z^{k}+(2-n) z^{k-1}+(2-n) z^{k-2}+\ldots+(2-n) z^{2}+(2-n) z+1} .
$$


The cases $n=4 g, k=2 g$ (orientable, genus $g$ ) and $n=2 g, k=g$ (non-orientable, genus $g$ ), corresponding to (torsion-free) surface groups with one vertex, were first computed by Cannon [6].

When $p=2 k+1$ there are $2 k$ types. For $i \in\{1, \ldots, k\}$, an element $g \in G$ with $|g|$ $=\mathrm{r} \geqq 1$ has type $t(g)=t_{i}$ if $g D \cap B(r-1)$ is a single edge, with one vertex of angle $2 \pi /(2 k+1)$ in $B(r-1)$ and the other vertex of angle $(2 i-1) 2 \pi /(2 k+1)$. For $i \in\{k+1, \ldots, 2 k-1\}, t(g)=t_{i}$ if $g D \cap B(r-1)$ is a single edge, with one vertex of angle $2 \pi /(2 k+1)$ and the other of angle $4 \pi(i-k) /(2 k+1)$. Finally, $g$ has type $t_{2 k}$ if $g D \cap B(r-1)$ is two edges with a vertex in common, the exterior vertices of $g D$ $\cap B(r-1)$ each have angle $2 \pi /(2 k+1)$ in $B(r-1)$, and the interior vertex has angle $4 k \pi /(2 k+1)$ in $B(r-1)$. An argument like the above shows that this gives a recursive structure. The initial vector and recursion matrix are the same as the comparable size matrices for the case $p=2 k$, except that in this case $A_{1 k}=n-4$. The growth function

$f(z)=\frac{z^{2 k}+2 z^{2 k-1}+\ldots+2 z^{k+1}+4 z^{k}+2 z^{k-1}+\ldots+2 z+1}{z^{2 k}+(2-n) z^{2 k-1}+\ldots+(2-n) z^{k+1}+(4-n) z^{k}+(2-n) z^{k-1}+\ldots+(2-n) z+1}$

In all of the above cases, the recursive structure is geometric and the recursion matrix A has I-A invertible. By Theorem 1, we know (without explicitly checking) that $f(1)=1 / \chi(G)$ in all of these examples. Note that the zeros of $f$ are not all roots of unity, in contrast to the Coxeter groups [3] or the examples in [6].

We now give an example of a recursive structure for a surface group in which I-A is not invertible. Let $G$ be the fundamental group of a closed orientable surface of genus 2 , and $\Sigma$ a finite generating set for $G$ coming from edge-pairing elements of a 16-gon whose image in the surface is shown in Fig. 26. By Poincarés Theorem, we can assume that $G$ is represented as a group of isometries of $\mathbb{H}^{2}$ and that $\Sigma$ is a geometric generating set for $G$ with respect to a Dirichlet region $D$ for $G$ in which each vertex has angle $2 \pi / 3$ or $\pi / 2$. From Fig. 26 , one sees that the angles alternate in the cycle $(2 \pi / 32 \pi / 32 \pi / 3 \pi / 2)$. Let $r \geqq 1$, and $g \in G$ with $|g|=r$. Then $g$ is of type $t_{1}$ if $g D \cap B(r-1)$ is a single edge $e$, and both vertices of $\partial$ e have angle $2 \pi / 3$ in $B(r-1)$. For $i \in\{2,3\}, t(g)=t_{i}$ if $g D \cap B(r-1)$ is a single edge $e$, one vertex of $\partial e$ has angle $2 \pi / 3$ in $B(r-1)$, and the other vertex has angle $\pi(i-1) / 2$ in $B(r-1)$. If $g D$ $\cap B(r-1)$ is two edges with a vertex in common, then $t(g) \in\left\{t_{4}, t_{5}, t_{6}\right\}$. The type is $t_{4}$ if the interior vertex of $g D \cap B(r-1)$ has angle $4 \pi / 3$, one exterior vertex has angle $2 \pi / 3$, and the other exterior vertex has angle $\pi / 2$. The type is $t_{5}$ if the interior vertex has angle $3 \pi / 2$ and each exterior vertex has angle $2 \pi / 3$, and $t(g)=t_{6}$ if the interior vertex has angle $4 \pi / 3$ and each exterior vertex has angle $2 \pi / 3$. An argument like the above shows that $\left\{t_{1}, \ldots, t_{6}\right\}$ gives a geometric recursive structure for $(G, \Sigma)$ with initial vector

$$
\mathbf{v}=\left[\begin{array}{l}
8 \\
8 \\
0 \\
0 \\
0 \\
0
\end{array}\right]
$$




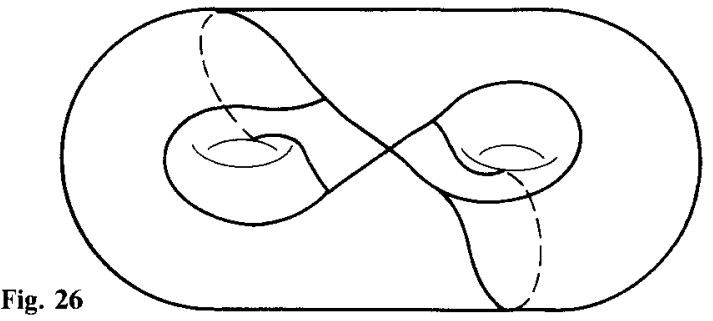

Fig. 26

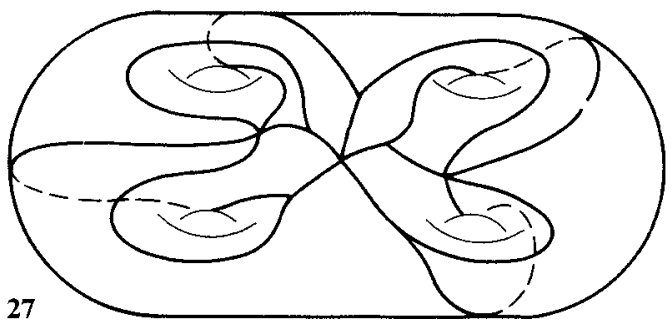

and recursion matrix

$$
\mathbf{A}=\left[\begin{array}{cccccc}
6 & 6 & 5 & 6 & 4 & 6 \\
5 & 6 & 6 & 5 & 6 & 4 \\
1 & 0 & 0 & 0 & 0 & 2 \\
1 / 2 & 1 / 2 & 1 / 2 & 1 / 2 & 1 & 0 \\
0 & 1 / 2 & 0 & 1 / 2 & 0 & 0 \\
0 & 0 & 1 / 2 & 0 & 0 & 0
\end{array}\right]
$$

Thus the growth function

$$
f(z)=\frac{z^{4}+5 z^{3}+8 z^{2}+5 z+1}{z^{4}-11 z^{3}-20 z^{2}-11 z+1} .
$$

We note that even though $\mathbf{I}-\mathbf{A}$ is not invertible, we still find $f(1)=1 / \chi$. In this case, $\mathbf{v}$ is in the image of $\mathbf{I}-\mathbf{A}$, say $(\mathbf{I}-\mathbf{A}) \mathbf{v}_{1}=\mathbf{v}$. It is easy to rewrite the formula of Theorem 1 to give

$$
f(z)=1 / \chi+(1 / 2 \chi)\left[(1-z) \mathbf{w} \mathbf{v}_{1}-(1-z)^{2} \mathbf{w}(\mathbf{I}-z \mathbf{A})^{-1} \mathbf{A} \mathbf{v}_{1}\right]
$$

Since 1 has multiplicity 1 as an eigenvalue of $A$, we find $f(1)=1 / \chi$.

Finally, we give an example in which $\mathbf{I}-\mathbf{A}$ is not invertible and $f(1) \neq 1 / \chi$. Let $G$ be the fundamental group of a closed orientable surface of genus 4 , and $\Sigma$ a finite generating set for $G$ determined by the edge-pairing elements of a 30-gon whose image in the surface is shown in Fig. 27. By Poincaré's Theorem, we can represent $G$ as a group of isometries of $\mathbb{H}^{2}$ and find a basepoint $x \in \mathbb{H}^{2}$ so that $\Sigma$ is a geometric generating set with respect to the Dirichlet region $D=D_{x}$, where $D$ has 30 sides of equal length, and the angles of $D$ strictly alternate between $2 \pi / 3$ and 
$2 \pi / 5$. We will give a geometric recursive structure $\left\{t_{1}, \ldots, t_{5}\right\}$ for $(G, \Sigma)$. Let $g \in G$ with $|g|=r \geqq 1$. For $i \in\{1,2,3\}, t(g)=t_{i}$ if $g D \cap B(r-1)$ is a single edge $e$, one vertex of $\partial e$ has angle $2 \pi / 3$ in $B(r-1)$, and the other vertex of $\partial e$ has angle $2 \pi i / 5$ in $B(r-1)$. The type of $g$ is $t_{4}$ if $g D \cap B(r-1)$ is two edges with a vertex in common, the interior vertex has angle $8 \pi / 5$ in $B(r-1)$ and each exterior vertex has angle $2 \pi / 3$ in $B(r-1)$. The type of $g$ is $t_{5}$ if $g D \cap B(r-1)$ is two edges with a vertex in common, the interior vertex has angle $4 \pi / 3$ in $B(r-1)$, and each exterior vertex has angle $2 \pi / 5$ in $B(r-1)$. An argument like the above shows that this gives a geometric recursive structure with initial vector

$$
\mathbf{v}=\left[\begin{array}{c}
30 \\
0 \\
0 \\
0 \\
0
\end{array}\right]
$$

and recursion matrix

$$
A=\left[\begin{array}{ccccc}
26 & 26 & 25 & 24 & 26 \\
1 & 1 & 1 & 2 & 0 \\
1 & 0 & 0 & 0 & 2 \\
0 & 1 / 2 & 0 & 0 & 0 \\
0 & 0 & 1 / 2 & 0 & 0
\end{array}\right]
$$

By Lemma 4, the growth function for $G$ with respect to $\Sigma$ is

$$
f(z)=\frac{z^{4}+4 z^{3}+7 z^{2}+4 z+1}{z^{4}-26 z^{3}-53 z^{2}-26 z+1} .
$$

Note that I $\mathbf{A}$ is not invertible, and $f(1)=-17 / 103 \neq-1 / 6=1 / \chi$. So even for geometric generating sets for surface groups, it is not always true that $f(1)=1 / \chi$.

\section{§4. Higher dimensions and open questions}

We would like to explain briefly why Theorem 1 should have an analogue for evendimensional co-finite volume hyperbolic groups. The two ingredients in the proof of Theorem 1 are:

(1) that $B(n)$ is a ball, and

(2) the Gauss-Bonnet formula, expressing area $(B(n))$ in terms of combinatorial data for $\partial B(n)$.

As far as (1) is concerned, nothing seems to be known in higher dimensions, except for Coxeter groups with the standard generating set, in which case $B(n)$ is a ball [8]. However, the generalized Gauss-Bonnet theorem does give an analogue for (2). For polyhedra in $\mathbb{H}^{n}$, this formula was known to Dehn [9], Klein [13, pp. 204-205], and certainly others. 
To describe the formula, let $B$ (a topological ball) be a polyhedron in $\mathbb{H}^{n}, n$ even. Then $\partial B$ is a union of lower-dimensional faces $B_{\lambda}^{i}, 0 \leqq i \leqq n-1,1 \leqq \lambda \leqq m_{i}$, defined by the intersections of $n-i$ hyperplanes. Each face $B_{\lambda}^{i}$ has an "inner angle" $\alpha_{\lambda}^{i}$, $0 \leqq \alpha_{\lambda}^{i} \leqq 1$, defined as follows: Let $x \in B_{\lambda}^{i}$. The collection of unit tangent vectors to $x$ which are normal to $B_{\lambda}^{i}$ forms an $(n-i-1)$-dimensional sphere $S^{n-i-1}$, and $\alpha_{\lambda}^{i}$ is the fraction of $S^{n-i-1}$ which points into $B$. This is independent of $x$. For example, the unique codimension zero face ( $B$ itself) has $\alpha^{n}=1$, a codimension one face has $\alpha_{\lambda}^{n-1}=1 / 2$, and a codimension two face has $\alpha_{\lambda}^{n-2}=\theta_{\lambda} / 2 \pi$, where $\theta_{\lambda}$ is the dihedral angle formed by the intersection of two hyperplanes. The Gauss-Bonnet formula states:

$$
\operatorname{volume}(B)=(-1)^{n / 2} \frac{K_{n}}{2}\left[1+\sum_{i=0}^{n-1}(-1)^{i} \sum_{\lambda=1}^{m} \alpha_{\lambda}^{i}\right],
$$

where $K_{n}=\frac{2^{n}\left(\frac{n}{2}-1\right) ! \pi^{n / 2}}{(n-1) !}$ is the volume of the unit $n$-sphere.

Now let $G$ be a discrete group of isometries of $\mathbb{H}^{n}$, with finite volume, and let $D$ be a Dirichlet region. By Cannon [5], the elements of $G$ fall into finitely many "cone types", and we have a recursion matrix A and initial vector $\mathbf{v}$ which yield the growth function as in $\S 1$. Each type is determined by local combinatorial data, in particular the way that a copy of $D$ corresponding to an element of length $n$, and a fixed type, is attached to $B(n-1)$, hence each element of length $n$ and a fixed type accounts for fixed angle contributions (of all codimensions $\geqq 1$ ) in $\partial B(n-1)$. Thus, as in $\S 2$ there is an attaching vector $w$ so that

$$
\mathbf{w A}^{n} \mathbf{v}=(-1)^{n / 2} \frac{K_{n}}{2} \sum_{i=0}^{n-1}(-1)^{i} \sum_{\lambda} \alpha_{\lambda}^{i}=\operatorname{vol}(B(n))-(-1)^{n / 2} \frac{K_{n}}{2} .
$$

(Here we are assuming $B(n)$ is a ball.) But

$$
\operatorname{vol}(B(n))=b_{n} \operatorname{vol}(D)=b_{n}(-1)^{n / 2} \frac{K_{n}}{2} \chi(G),
$$

by the Gauss-Bonnet formula for $\mathbb{H}^{n} / G$. Hence

$$
b_{n}=1 / \chi\left[1+(-1)^{n / 2} \frac{2}{K_{n}} \mathbf{w A}^{n} \mathbf{v}\right] \text {. }
$$

As in Theorem 1, we find

$$
f(z)=1 / \chi+(-1)^{n / 2} \frac{2(1-z)}{K_{n} \chi}\left(\mathbf{w},(\mathbf{I}-z \mathbf{A})^{-1} \mathbf{v}\right),
$$

so one again expects (generically) that $f(1)=1 / \chi$.

In our view, the main open problems along the lines of this work are:

(1) Understand the "error term" $f(1)-1 / \chi$ when it is non-zero. In $\S 3$ we gave one example - the fundamental group of a closed, orientable surface of genus 4 , 
and $D$ is a 30 -sided polygon whose angles alternate between $2 \pi / 3$ and $2 \pi / 5$. This is just one example of an infinite family. We have computed growth functions for all tilings of $\mathrm{H}^{2}$ by even-sided polygons whose angles alternate between $2 \pi / p$ and $2 \pi / q$. It turns out that the "error" is non-zero whenever both $p$ and $q$ are odd. We will describe these results elsewhere.

(2) Understand the symmetries of the growth functions. Notice that all of the examples in $\S 3$ give reciprocal functions: $f(z)=f(1 / z)$. This symmetry in the coefficients of the numerator and denominator of $f(z)$ seems to be the rule. Actually, we do know of two classes of counterexamples - one class is the $(3,3, r)$ triangle groups with 4-sided Dirichlet domains, which were already singled out in $\S 1$ as exceptional. In these cases, the denominators of $f(z)$ have symmetric coefficients, but not the numerators. Based on many computations, we are tempted to conjecture that for cocompact Fuchsian groups the denominators always have symmetric coefficients.

(3) Understand the poles of $f(z)$. This is closely related to (2), see Cannon [6]. How does the radius of convergence of $g(z)(=$ the smallest modulus of the poles of $f(z))$ change as the geometry of $D$ changes? Can $f(z)$ have a pole at 1 ? While $f(z)$ often has only one pole in the open unit disk, there are examples for which $f(z)$ has more than one pole in the open disk. If $f(z)$ has only one pole in the open disk and the denominator has symmetric coefficients, then this pole is the reciprocal of a Salem number.

(4) Compute some higher dimensional examples. Some three-dimensional examples have been computed by Cannon and by Grayson (see [10]).

\section{Note added in proof.}

For cocompact planar groups with geometric generating sets, the authors have shown that $f(z)=f(1 / 2)$ except for the two classes of counterexamples mentioned above. The proof will appear in a paper titled Symmetries of planar growth functions.

\section{References}

1. Bass, H.: The degree of polynomial growth of finitely generated nilpotent groups. Proc. Lond. Math. Soc. 25, 603-614 (1972)

2. Benson, M.: Growth Series of finite extension of $\mathbb{Z}^{n}$ are rational. Invent. math. 73, 251-269 (1983)

3. Bourbaki, N.: Groupes et algèbres de Lie. Chapitres 4, 5 et 6. Paris: Hermann 1968

4. Brenner, J.L., Lyndon, R.C.: Infinite Eulerian tessellations. Discrete Math. 46, 111-132 (1983)

5. Cannon, J.W.: The combinatorial structure of cocompact discrete hyperbolic groups. Geom. Dedicata 16, 123-148 (1984)

6. Cannon, J.W.: The growth of the closed surface groups and the compact hyperbolic Coxeter groups. Preprint (1983)

7. Cannon, J.W.: Colored graphs. Preprint (1983)

8. Davis, M.: Groups generated by reflections and aspherical manifolds not covered by Euclidean space. Ann. Math. 117, 293-324 (1983)

9. Dehn, M.: Die Eulersche Formel im Zusammenhang mit dem Inhalt in der Nicht-Euklidischen Geometrie. Math. Ann. 61, 561-586 (1905)

10. Grayson, M.A.: Geometry and growth in three dimensions. Ph.D. thesis. Princeton University (1983)

11. Grigorchuk, R.I.: On Milnor's problem of group growth. Sov. Math., Dokl. 28, 23-26 (1983)

12. Gromov, M.: Groups of polynomial growth and expanding maps. Publ. Math., Inst. Hautes Etud. Sci. 53, 53-78 (1981) 
13. Klein, F.: Vorlesungen über Nicht-Euklidische Geometrie. Die Grundlehren der Mathematischen Wissenschaften, Vol. 26. Berlin-Göttingen-Heidelberg: Springer 1928

14. Maskit, B.: On Poincaré's theorem for fundamental polygons. Adv. Math. 7, 219-230 (1971)

15. Milnor, J.: Problem. Amer. Math. Monthly 75, 685-686 (1968)

16. Milnor, J.: A note on curvature and fundamental groups. J. Differ. Geom. 2, 1-7 (1968)

17. Milnor, J.: Growth of finitely generated solvable groups. J. Differ. Geom. 2, 447-449 (1968)

18. Parry, W.: Counterexamples involving growth series and the Euler characteristic. Proc. Amer. Math. Soc. (to appear)

19. Poincaré, H.: Théorie des groupes fuchsiens. Acta Math. 1, 1-62 (1882)

20. Serre, J-P.: Cohomologie des groups discrets, in Prospects in Mathematics. Ann. Math. Stud. No 70 , Princeton, 1971

21. Smythe, N.: Growth funetions and Euler series. Invent. math. 77, 517-531 (1984)

22. Wagreich, P.: The growth function of a discrete group. Proc. Conference on Algebraic Varieties with Group Actions. Lecture Notes, Vol, 956, pp. 125-144 Berlin-Heidelberg-New York: Springer 1982

23. Wolf, J.A.: Growth of finitely generated groups and curvature of Riemannian manifolds. J. Differ. Geom. 2, 421-446 (1968) 